\title{
Measurement of the pressure dependence of air fluorescence emission induced by electrons
}

\author{
AIRFLY Collaboration: M. Ave ${ }^{a}$, M. Bohacova ${ }^{\text {, }}$, \\ B. Buonomo ${ }^{\mathrm{c}}$, N. Busca ${ }^{\mathrm{a}}$, L. Cazon ${ }^{\mathrm{a}}$, S.D. Chemerisov ${ }^{\mathrm{d}}$, \\ M.E. Conde ${ }^{d}$, R.A. Crowell ${ }^{d}$, P. Di Carlo ${ }^{\mathrm{e}}$, C. Di Giulio ${ }^{\mathrm{f}}$, \\ M. Doubrava ${ }^{g}$, A. Esposito ${ }^{c}$, P. Facal ${ }^{\text {h }}$, F.J. Franchini ${ }^{\mathrm{d}}$, \\ J. Hörandel ${ }^{\mathrm{i}}$, M. Hrabovsky ${ }^{\mathrm{b}}$, M. Iarlori ${ }^{\mathrm{e}}$, T.E. Kasprzyk ${ }^{\mathrm{d}}$, \\ B. Keilhauer ${ }^{\mathrm{i}}$, H. Klages ${ }^{\mathrm{j}}$, M. Kleifges ${ }^{\mathrm{k}}$, S. Kuhlmann ${ }^{\mathrm{d}}$, \\ G. Mazzitelli ${ }^{\text {c }}$, L. Nozka ${ }^{\text {b }}$, A. Obermeier ${ }^{\mathrm{i}}$, M. Palatka ${ }^{\mathrm{b}}$, \\ S. Petrera ${ }^{\mathrm{e}}$, P. Privitera ${ }^{\mathrm{f}, *}$, J. Ridky ${ }^{\mathrm{b}}$, V. Rizi ${ }^{\mathrm{e}}$, \\ G. Rodriguez ${ }^{f}$, F. Salamida ${ }^{e}$, P. Schovanek ${ }^{b}$, H. Spinka ${ }^{\text {, }}$, \\ E. Strazzeri ${ }^{\mathrm{f}}$, A. Ulrich $^{\ell}$, Z.M. Yusof ${ }^{\mathrm{d}}$, V. Vacek ${ }^{\mathrm{g}}$, \\ P. Valente ${ }^{\mathrm{m}}$, V. Verzi ${ }^{\mathrm{f}}$, T. Waldenmaier ${ }^{\mathrm{j}}$ \\ a University of Chicago, Enrico Fermi Institute, 5640 S. Ellis Ave., Chicago, IL \\ 60637, United States \\ ${ }^{\mathrm{b}}$ Institute of Physics of the Academy of Sciences of the Czech Republic, Na \\ Slovance 2, CZ-182 21 Praha 8, Czech Republic \\ ${ }^{\mathrm{c}}$ Laboratori Nazionali di Frascati dell'INFN, INFN, Sezione di Frascati, Via \\ Enrico Fermi 40, Frascati, Rome 00044, Italy \\ d Argonne National Laboratory, Argonne, IL 60439 United States

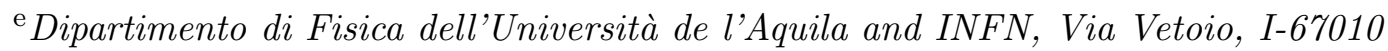 \\ Coppito, Aquila, Italy \\ ${ }^{\mathrm{f}}$ Dipartimento di Fisica dell'Università di Roma Tor Vergata and Sezione INFN, \\ Via della Ricerca Scientifica, I-00133 Roma, Italy \\ ${ }^{\mathrm{g}}$ Czech Technical University, Technicka 4, 16607 Praha 6, Czech Republik \\ h Departamento de Física de Partículas, Campus Sur, Universidad, E-15782 \\ Santiago de Compostela, Spain

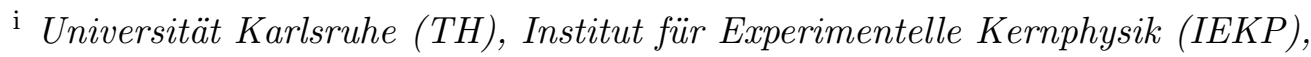 \\ Postfach 6980, D - 76128 Karlsruhe, Germany \\ j Forschungszentrum Karlsruhe, Institut für Kernphysik, Postfach 3640, D - 76021 \\ Karlsruhe, Germany \\ ${ }^{\mathrm{k}}$ Forschungszentrum Karlsruhe, Institut für Prozessdatenverarbeitung und \\ Elektronik, Postfach 3640, D - 76021 Karlsruhe, Germany \\ ${ }^{\ell}$ Physik Department E12, Technische Universität Muenchen, James Franck Str. 1, \\ D-85748 Garching, Germany \\ ${ }^{\mathrm{m}}$ Sezione INFN di Roma 1, Ple. A. Moro 2, I-00185 Roma, Italy
}




\begin{abstract}
The fluorescence detection of ultra high energy $\left(\gtrsim 10^{18} \mathrm{eV}\right)$ cosmic rays requires a detailed knowledge of the fluorescence light emission from nitrogen molecules, which are excited by the cosmic ray shower particles along their path in the atmosphere. We have made a precise measurement of the fluorescence light spectrum excited by $\mathrm{MeV}$ electrons in dry air. We measured the relative intensities of 34 fluorescence bands in the wavelength range from 284 to $429 \mathrm{~nm}$ with a high resolution spectrograph. The pressure dependence of the fluorescence spectrum was also measured from a few $\mathrm{hPa}$ up to atmospheric pressure. Relative intensities and collisional quenching reference pressures for bands due to transitions from a common upper level were found in agreement with theoretical expectations. The presence of argon in air was found to have a negligible effect on the fluorescence yield. We estimated that the systematic uncertainty on the cosmic ray shower energy due to the pressure dependence of the fluorescence spectrum is reduced to a level of $1 \%$ by the AIRFLY results presented in this paper.
\end{abstract}

Key words: Air Fluorescence Detection, Ultra High Energy Cosmic Rays, Nitrogen Collisional Quenching PACS: , 96.50.S-, 96.50.sb, 96.50.sd, 32.50.+d, 33.50.-j, 34.50.Fa, 34.50.Gb

\title{
1 Introduction
}

The detection of ultra high energy $\left(\gtrsim 10^{18} \mathrm{eV}\right)$ cosmic rays using nitrogen fluorescence light emission from extensive air showers (EAS) is a well established technique, used by the Fly's Eye [1], HiRes [2], and Pierre Auger Observatory [3] experiments, and planned for the Telescope Array [4], which is presently under construction. It has also been proposed for the satellite-based EUSO [5] and OWL [6] projects. Excitation of atmospheric nitrogen by EAS charged particles induces fluorescence emission, mostly in the wavelength range between 300 to $430 \mathrm{~nm}$. Information on the longitudinal EAS development can be obtained by fluorescence telescopes by recording the light intensity as a function of time and incoming direction; this information is related to the primary cosmic ray energy and type. However, the fluorescence light yield from EAS charged particles must be well known at each point within the shower, and corrections applied for atmospheric effects between the shower and the telescope for an accurate primary energy determination. Thus, the intensities of the fluorescence bands should be measured over a range of air pressure and

\footnotetext{
* corresponding author

Email address: priviter@roma2.infn.it ( P. Privitera).
} 
temperature corresponding to altitudes up to several tens of $\mathrm{km}$, the typical elevation of EAS development in the atmosphere.

A number of other experiments have made measurements of the fluorescence light yield pertinent to EAS. These include early low-energy stopped-particle results in air by Bunner [7] and electrons in air by Davidson and O'Neil [8]. More recently, Kakimoto et al. [9] obtained the light yields with three narrow and one broad band optical filters with electrons from a radioactive source at 1.4 MeV and from a synchrotron at 300, 650, and $1000 \mathrm{MeV}$ in dry air and pure nitrogen. Nagano et al. [10] measured the light yields in pure nitrogen and dry air with electrons of average energy $0.85 \mathrm{MeV}$ from a radioactive source, using 14 narrow-band filters. Belz et al. [11] used 28.5 GeV electrons in pure nitrogen and dry air to measure the light yield through a HiRes broad band optical filter ( 300-400 nm) as a function of pressure. Colin et al. [12] measured the fluorescence light yield with a broad band optical filter at electron energies of $1.5 \mathrm{MeV}, 20 \mathrm{GeV}$ and $50 \mathrm{GeV}$. The application of these fluorescence measurements to EAS experiments has been described recently by de Souza et al. [13], Keilhauer et al. [14, and Arqueros et al. [15].

The uncertainty on the fluorescence light yield is currently one of the main systematic uncertainties on the cosmic ray energy determination by EAS experiments which employ the fluorescence technique. The absolute fluorescence yield is known only at the level of $15 \%$ and for a few electron energies. Recent spectral measurements are limited by the use of optical filters, while the early data of Bunner are based on measurements with coarse spectral resolution and large systematic uncertainties. The data reported here are part of a program by the AIRFLY (AIR FLuorescence Yield) collaboration to measure the fluorescence light yield with significantly improved precision over the electron kinetic energy range from $\mathrm{keV}$ to $\mathrm{GeV}$ using several accelerators. This paper describes the measurement of the pressure dependence of the relative yields of the fluorescence bands in the wavelength range $284-429 \mathrm{~nm}$. The absolute yield, as well as the temperature and humidity dependence of the fluorescence spectrum, are currently being analysed, and will be reported elsewhere.

The relative intensities of 34 fluorescence bands over the wavelength range 284 - $429 \mathrm{~nm}$ were measured in dry air at $800 \mathrm{hPa}$ with a high resolution spectrograph. The pressure dependence of the fluorescence spectrum was also studied from a few hPa up to atmospheric pressure. The high beam current needed for a measurement of the fluorescence spectrum was provided by a DC beam of $3 \mathrm{MeV}$ electrons. The pressure dependence of the $337 \mathrm{~nm}$ band in dry air was measured in a different set-up using a narrow band optical filter and a photomultiplier tube. We used a $14 \mathrm{MeV}$ electron beam for this measurement, which had the advantage of much better stability of the beam position, a small spot size and reduced multiple scattering effects compared to the $3 \mathrm{MeV}$ beam. These beam characteristics were important to reduce 
the systematic uncertainties. We measured the collisional quenching reference pressure of the $337 \mathrm{~nm}$ band, $p_{\text {air }}^{\prime}(337)$, by studying the ratio of fluorescence emission in nitrogen and air. With this method, we eliminated the bias from undetected light due to secondary electrons escaping the detector's field of view at low pressures. The collisional quenching reference pressures of the other fluorescence bands were obtained from the pressure dependence of their relative intensities, using $p_{\text {air }}^{\prime}(337)$ as normalization.

This paper is organized as follows. Section 2 presents equations used to parameterize the pressure dependence of the fluorescence light yield. The experimental method and hardware are given in Section 3. The measured fluorescence spectrum at $800 \mathrm{hPa}$ in dry air, the method used to estimate the band intensities, and a comparison to theoretically expected intensities are discussed in Section 4. The $337 \mathrm{~nm}$ pressure dependence measurements at $14 \mathrm{MeV}$ are given in Section 5, as well as a new analysis of the data which minimize the systematic uncertainties due to the spatial distribution of the fluorescence emission induced by secondary electrons. The measurement of the pressure dependence of the remaining spectral bands is presented in Section 6. Section 7 presents applications to EAS, and Section 8 summarizes this work.

\section{Fluorescence yield}

Electrons passing through air excite the nitrogen molecules, directly as well as through secondary electrons produced along the path in the gas. Nitrogen deexcitation results in a fluorescence spectrum which, in the range 300 to $430 \mathrm{~nm}$, consists mainly of transitions from the so-called second positive system (2P) of molecular nitrogen $\mathrm{N}_{2}$ and the first negative system $(1 \mathrm{~N})$ of ionised nitrogen molecules $\mathrm{N}_{2}^{+}$. Throughout the paper, the notations $2 \mathrm{P}\left(v, v^{\prime}\right)$ and $1 \mathrm{~N}\left(v, v^{\prime}\right)$, corresponding to $C^{3} \Pi_{u}(v) \rightarrow B^{3} \Pi_{g}\left(v^{\prime}\right)$ and $B^{2} \Sigma_{u}^{+}(v) \rightarrow X^{2} \Sigma_{g}^{+}\left(v^{\prime}\right)$ [16], will be used to indentify the relevant electronic-vibrational transitions. Not all the excited nitrogen molecules emit fluorescence photons, since they may transfer their energy to other molecules through collision. This quenching process introduces a dependence of the fluorescence emission on the gas pressure and temperature, because the collisional rate depends on the average separation distance and velocity of the molecules.

The process of fluorescence emission induced by electrons in nitrogen gas can be described in terms of fluorescence efficiency [7] [10], namely the ratio of the energy emitted by the excited gas in fluorescence photons to the energy deposited in the gas by the electron. The fluorescence efficiency of photons of 
wavelength $\lambda$ at a given nitrogen pressure $p$ is usually parameterized as:

$$
\Phi_{\mathrm{N}_{2}}(\lambda, p)=\frac{\Phi_{\mathrm{N}_{2}}^{0}(\lambda)}{1+\frac{p}{p_{\mathrm{N}_{2}}^{\prime}(\lambda)}}
$$

where $p_{\mathrm{N}_{2}}^{\prime}(\lambda)$ is the collisional quenching reference pressure, and $\Phi_{\mathrm{N}_{2}}^{0}(\lambda)$ is the fluorescence efficiency in absence of collisional quenching (in the limit $p \rightarrow 0$ the distance between nitrogen molecules becomes very large and they cannot de-excite by collisions). Here, the fluorescence yield is defined as the ratio of the fluorescence efficiency to the photon energy $E_{\lambda}$, that is the number of photons emitted by the excited gas per energy deposited by the electron:

$$
Y_{\mathrm{N}_{2}}(\lambda, p)=\frac{Y_{\mathrm{N}_{2}}^{0}(\lambda)}{1+\frac{p}{p_{\mathrm{N}_{2}}^{\prime}(\lambda)}}
$$

where $Y_{\mathrm{N}_{2}}^{0}(\lambda)=\Phi_{\mathrm{N}_{2}}^{0}(\lambda) / E_{\lambda}$.

The quenching reference pressure for nitrogen can be written in terms of the lifetime of the excited state to decay to any lower state $\tau_{0}$ and the cross section for nitrogen-nitrogen collisional de-excitation $\sigma_{\mathrm{NN}}$ [7][10]:

$$
\frac{1}{p_{\mathrm{N}_{2}}^{\prime}}=\frac{4 \tau_{0}}{\sqrt{\pi M_{\mathrm{N}} k T}} \sigma_{\mathrm{NN}}
$$

where $M_{\mathrm{N}}$ is the $\mathrm{N}_{2}$ molecular mass, $k$ is Boltzmann's constant, and $T$ is temperature.

The fluorescence yield in air is given by:

$$
Y_{\text {air }}(\lambda, p)=\frac{Y_{\mathrm{N}_{2}}^{0}(\lambda) f_{\mathrm{N}_{2}}}{1+\frac{p}{p_{\text {air }}^{\prime}(\lambda)}}
$$

where $f_{\mathrm{N}_{2}}$ is the fraction of nitrogen molecules in air (79\%). The quenching reference pressure in air takes into account also nitrogen-oxygen collisional de-excitation:

$$
\frac{1}{p_{\text {air }}^{\prime}}=\frac{4 \tau_{0}}{\sqrt{\pi M_{\mathrm{N}} k T}}\left(f_{\mathrm{N}_{2}} \sigma_{\mathrm{NN}}+f_{\mathrm{O}_{2}} \sigma_{\mathrm{NO}} \sqrt{\frac{M_{\mathrm{N}}+M_{\mathrm{O}}}{2 M_{\mathrm{O}}}}\right)=\frac{f_{\mathrm{N}_{2}}}{p_{\mathrm{N}_{2}}^{\prime}}+\frac{f_{\mathrm{O}_{2}}}{p_{\mathrm{O}_{2}}^{\prime}},
$$

where $f_{\mathrm{O}_{2}}$ is the fraction of oxygen molecules in air $(21 \%), M_{\mathrm{O}}$ is the $\mathrm{O}_{2}$ molecular mass and $\sigma_{\mathrm{NO}}$ is the cross section for nitrogen-oxygen collisional de-excitation. In this paper, we will not interpret the measurements in terms 
of collisional cross sections, but rather use the quenching reference pressure for each band as a phenomenological description of the data. Note that the fluorescence yield process finds in Eqs. (41) - (5I) its simplest description. Mechanisms which may modify Eqs. (4)-(5) have been discussed in the literature (see for ex. 14] 17] [18]). On the other hand, it will be shown in the following Sections that the ansatz described by Eqs. (4)-(5) does indeed provide a good description of our data.

The typical arrangement in fluorescence experiments involves a photon detector, e.g. a photomultiplier, viewing a portion of gas volume $\left(\mathrm{N}_{2}\right.$ or air) along the electron path. The detected signal is thus proportional to the number of fluorescence photons $N_{\lambda}^{g a s}$ emitted in the detector field of view:

$$
N_{\lambda}^{g a s}=E_{d e p}^{g a s} Y_{g a s}(\lambda, p)
$$

where $E_{d e p}^{g a s}$ is the energy deposited by the electron in the gas volume viewed by the photon detector. The energy $E_{d e p}^{g a s}$ depends on the specific geometry of the experiment, and can be estimated by a detailed Monte Carlo simulation, including effects like multiple scattering and secondary electrons. Nevertheless, for a given experimental arrangement, we can write:

$$
E_{d e p}^{g a s}=\left(\frac{d E}{d X}\right)_{g a s} \rho_{g a s} D_{g a s}(p),
$$

where $(d E / d X)_{\text {gas }}$ is the collisional energy loss, $\rho_{\text {gas }}$ is the gas density and $D_{\text {gas }}(p)$ is an effective length which takes into account the specific experimental arrangement. Notice that a pressure dependence has been included in $D_{\text {gas }}(p)$. In fact, as the gas pressure goes down, an increasing fraction of secondary electrons escapes the detector field of view, and correspondingly part of the fluorescence emission is not detected. The net effect is a reduction of the effective length $D_{\text {gas }}$, which is thus expected to be a decreasing function of $p$.

The number of detected fluorescence photons can then be written:

$$
N_{\lambda}^{g a s}=\left(\frac{d E}{d X}\right)_{g a s} \frac{p}{R_{g a s} T} D_{g a s}(p) Y_{g a s}(\lambda, p),
$$

where $R_{\text {gas }}$ is the specific gas constant, $T$ is the gas temperature and the gas equation of state has been used. 


\section{Experimental method}

Measurements were performed at two Argonne National Laboratory accelerators. Spectra from 284 - $429 \mathrm{~nm}$ were recorded with $3 \mathrm{MeV}$ electrons in various gases and pressures using the Chemistry Division's Van de Graaff (VdG) electron accelerator [19] [20]. The relative light yield of the $337 \mathrm{~nm}$ band was measured at $14 \mathrm{MeV}$ at the Argonne Wakefield Accelerator (AWA) Facility [21] in dry air and pure nitrogen as a function of pressure. This relative light

yield, combined with the measured spectra, allowed the pressure dependence of the fluorescence bands to be derived.

\subsection{Van de Graaff Measurements}

The Van de Graaff accelerator is capable of accelerating electrons and protons to kinetic energies from 0.5 to $3 \mathrm{MeV}$, with beam currents in excess of $10 \mu \mathrm{A}$. The VdG can operate in DC current or pulsed mode. For the spectrum measurements it was operated in the DC current mode with typical beam currents of $\sim 10 \mu \mathrm{A}$, and nominal beam kinetic energy of $3.0 \mathrm{MeV}$. After exiting the $\mathrm{VdG}$, the electron beam was bent $30^{\circ}$ in an electromagnet and was focused near the exit window $2.00 \mathrm{~m}$ from the magnet center. The electrons left the accelerator vacuum through a $35 \mathrm{~mm}$ diameter, $0.152 \mathrm{~mm}$ thick dura-aluminum window; see Fig. 1. An additional quartz window was placed downstream of the aluminum window during beam tuning. The beam spot image from the quartz was reflected with a mirror to a camera. The beam spot size was typically $6 \mathrm{~mm}$ diameter, and a side-to-side beam motion of approximately $5 \mathrm{~mm}$ was observed due to small $(<1 \%)$ variations in the $\mathrm{VdG}$ energy on time scales of seconds. The quartz window and mirror were removed during measurements of the spectra.

The layout for the Van de Graaff measurements is shown in Fig. 1. The electrons entered the pressure chamber after $6 \mathrm{~cm}$ of air. Light produced in the gas was focused by an aluminum spherical mirror onto the end of a $10 \mathrm{~m}$ long, $1.5 \mathrm{~mm}$ diameter pure silica core optical fiber, which brought the light to a spectrograph. The optical fiber was placed outside the pressure chamber, and light reached the fiber end passing through a quartz window. The spectrograph

was located behind a concrete block wall and had additional lead shielding to protect it from the radiation produced by the $\mathrm{VdG}$. 


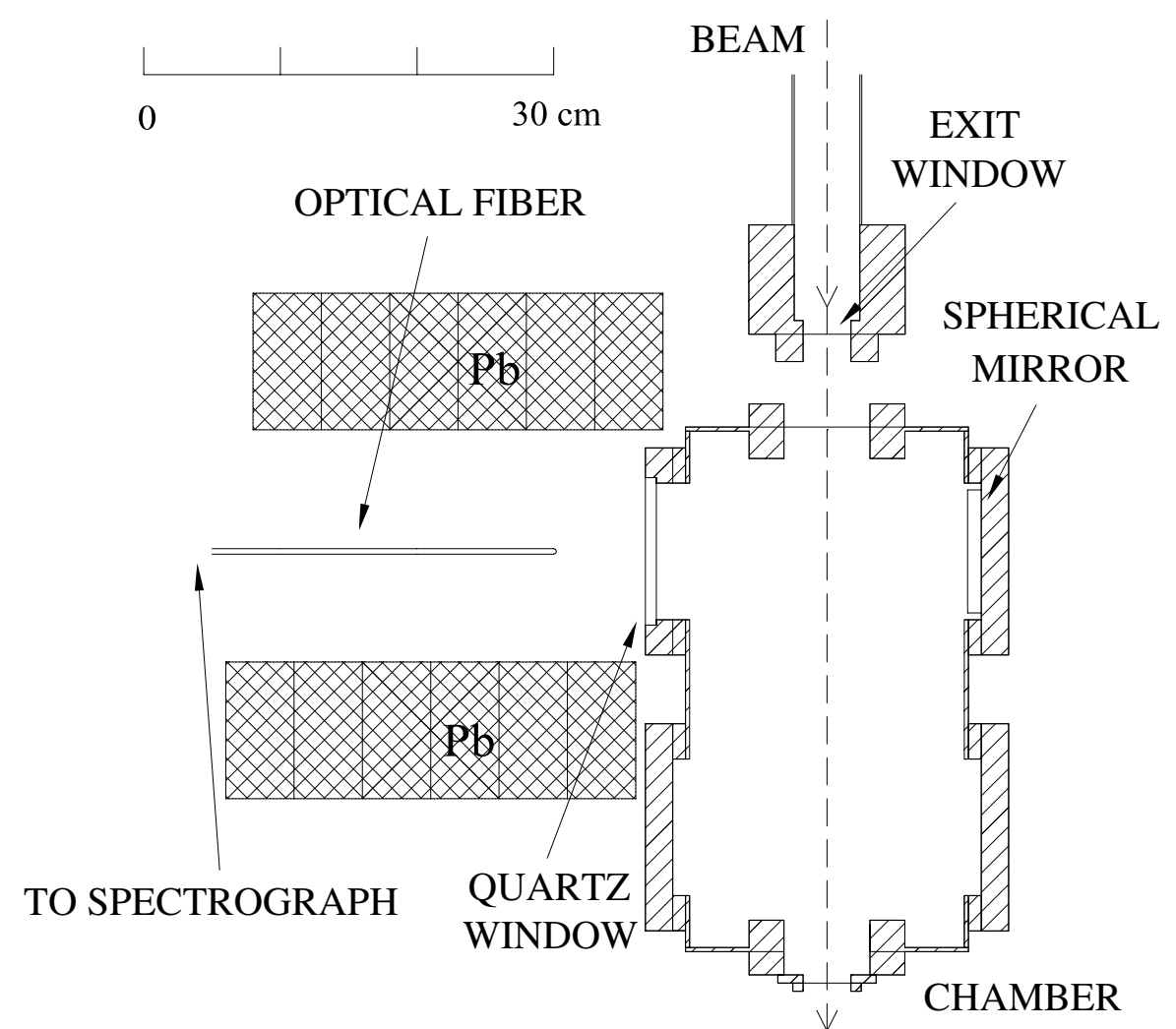

Fig. 1. Layout of the measurements at the Van de Graaff, including the exit window from the accelerator vacuum, the pressure chamber, spherical mirror, quartz window, and optical fiber.

\subsection{Argonne Wakefield Accelerator Measurements}

The Argonne Wakefield Accelerator can accelerate electrons to kinetic energies from $3 \mathrm{MeV}$ to $16 \mathrm{MeV}$. It was operated in the pulsed mode at $5 \mathrm{~Hz}$, with bunches of maximum charge of $\sim 1 \mathrm{nC}$ and length $\sim 15 \mathrm{ps}(\mathrm{FWHM})$. A new set of three quadrupole magnets and two Vernier steering dipole magnets was added for this experiment to focus and steer the beam. The nominal beam kinetic energy was $14.0 \mathrm{MeV}$, with an estimated energy spread of $\pm 0.3 \mathrm{MeV}$. The electrons exited the accelerator vacuum through a $32 \mathrm{~mm}$ diameter, $0.13 \mathrm{~mm}$ thick beryllium window; see Fig. 22. During beam tuning, the beam spot image from a quartz plate placed at $45^{\circ}$ to the beam direction near the exit window was viewed with a camera. The beam spot size was typically $5 \mathrm{~mm}$ diameter, with negligible beam motion. The quartz plate was removed during the fluorescence measurements.

The beam intensity was monitored with an integrating current transformer (ICT, [22]), immediately before the beam exit flange. The signal from the ICT was integrated, digitized, and recorded for each beam bunch. The ICT was 


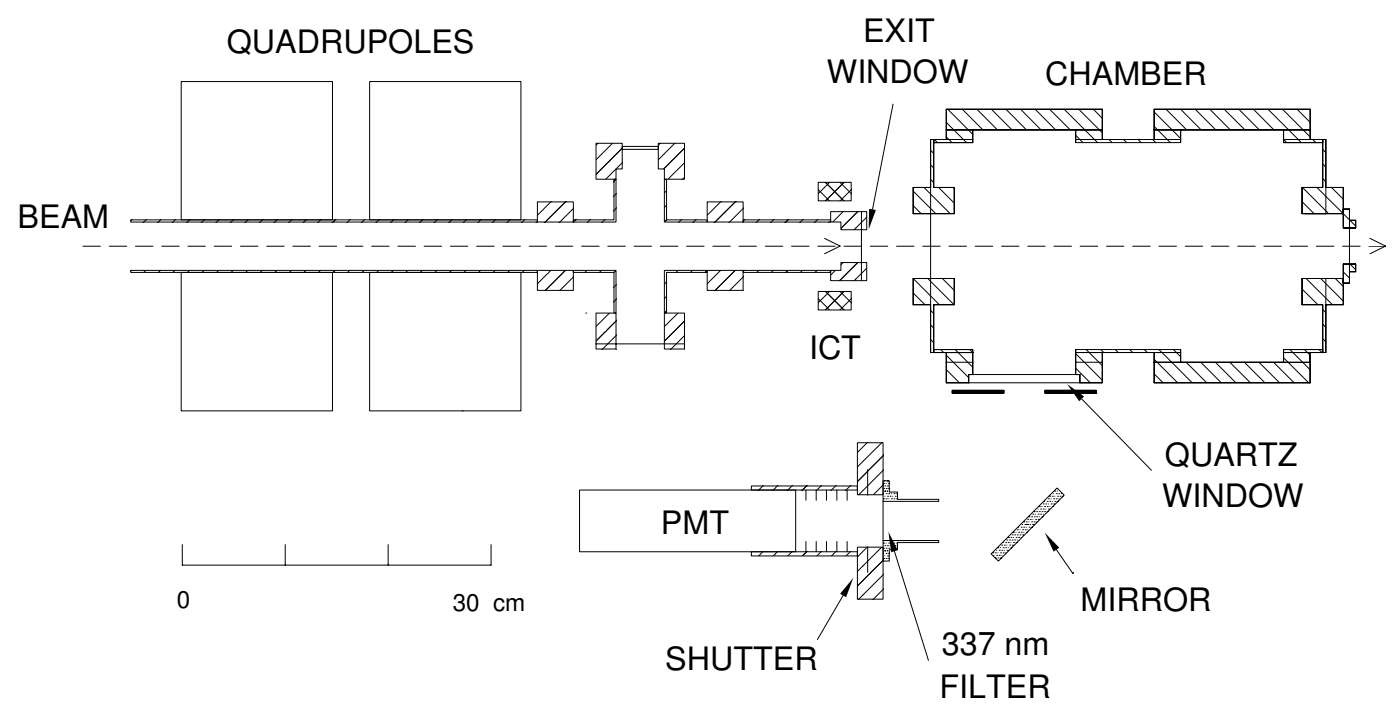

Fig. 2. Layout of the measurements at the Argonne Wakefield Accelerator, including the exit window from the accelerator vacuum, ICT, the pressure chamber, quartz window, mirror, $337 \mathrm{~nm}$ filter, mechanical shutter, and photomultiplier. The last two quadrupoles are also shown, but not the third quadrupole or the adjacent dipole. Considerable lead shielding of the photomultiplier and mirror were also omitted for clarity.

calibrated by the manufacturer to give an output charge 0.025 times the input charge.

The layout for the AWA measurements is shown in Fig. 2, including the last two quadrupoles and ICT. Light produced in the gas propagated out of the chamber through a quartz window, mechanical shutter, and $337 \mathrm{~nm}$ filter, and was reflected by a mirror to a photomultiplier. The shutter could be closed remotely to allow measurements of background. A Hamamatsu H7195P photomultiplier [23] was used. A second photomultiplier of the same type was used to monitor backgrounds. Both were surrounded by considerable lead shielding to reduce beam-related backgrounds. The first photomultiplier, mirror, filter, and shutter are also shown in Fig. 2 .

A VME data acquisition system was used. The signals from the photomultipliers and the ICT were integrated by a Lecroy model 1182 charge integrating ADC. The accelerator timing signal was used to produce the integrating gate of 200 ns width. Signals were recorded for each electron bunch passage in the pressure chamber. 


\subsection{Pressure Chamber}

The pressure chamber was constructed of an aluminum tube with various flanges welded to it for windows, gauges, gas inlet, and pump-out. The aluminum tube had an inner diameter of $201 \mathrm{~mm}$, length $378 \mathrm{~mm}$, and wall thickness $3 \mathrm{~mm}$. The exit window of $0.1 \mathrm{~mm}$ thick aluminum was bolted to the one end. The entrance window of $0.50 \mathrm{~mm}$ thick beryllium, $35 \times 55 \mathrm{~mm}$, was diffusion bonded to a Conflat flange. All vacuum seals were made with O-rings. A top view of the pressure chamber is shown in Fig. 1.

A remotely-controlled gas handling and vacuum system was used with the pressure chamber. A dry scroll vacuum pump [24] was used to reduce the pressure and evacuate the chamber. The chamber pressure was measured [25] at the pump-out port. Three types of high purity dry gases were used: $99.9995 \%$ pure nitrogen, a mixture with argon (78.0\% nitrogen, $21.0 \%$ oxygen, $1.0 \%$ argon), and a mixture without argon (79\% nitrogen, $21 \%$ oxygen).

\subsection{Spectrograph}

Air fluorescence spectra were recorded by an Oriel MS257 $7^{T M}$ spectrograph [26]. The main characteristics of the instrument include an asymmetrical Czerny-Turner design, F number equal to 3.9, and focal length of $220 \mathrm{~mm}$. A holographic grating with 1200 lines/mm and blaze wavelength of $250 \mathrm{~nm}$ was used. The light from the grating was collected by a $1024 \times 255$ CCD pixel array (Andor DV420 BU2 [27]). The CCD camera, of 26x26 $\mu \mathrm{m}$ pixel size, was back-illuminated and had a large quantum efficiency $(\sim 50 \%)$ in the 300 to $400 \mathrm{~nm}$ wavelength range. The linear dispersion of spectrograph, with the grating used, is $3.2 \mathrm{~nm} / \mathrm{mm}$, that gives a wavelength window of about $85 \mathrm{~nm}$. This means that the air fluorescence spectrum, which extends over more than $100 \mathrm{~nm}$, did not fit in a single wavelength window of the spectrograph. Thus, in a fluorescence run the spectrograph collected data in sequence, first in the range 284-369 $\mathrm{nm}$ and then in the range 344-429 $\mathrm{nm}$. For each wavelength range, 50 spectra of 1 second integration time were taken. The spectrograph operation and data acquisition was fully automated.

The spectral bands emitted by a mercury pencil lamp (Oriel no. 60635 [26]) in the region 290 to $410 \mathrm{~nm}$ were used to calibrate the spectral response of the spectrograph. The calibration was then refined by including the positions of the nitrogen emission bands as seen in the air fluorescence spectrum measurement.

A quartz tungsten halogen lamp (Oriel no. 63350 [26]) was used to calibrate the relative spectrograph sensitivity as a function of wavelength. The spectral 
irradiance of the lamp is traceable to NIST [28], and its uncertainty amounts to $2.5 \%$ in the wavelength range of interest for the air fluorescence measurement.

The spectrograph calibration was performed on the beam line, between air fluorescence measurements. A flange on the top of the pressure chamber allowed the insertion of the calibration lamp along the beam path. In order to take into account the spatial distribution of the air fluorescence emission in the experiment, the calibration lamp was placed in different positions along the beam axis and the recorded lamp spectra were appropriately combined. By comparison with the known lamp spectrum, calibration factors for the relative intensity as a function of wavelength were determined. The two spectrograph ranges used for the air fluorescence measurement, 284-369 nm and 344-429 nm, were independently calibrated. Their relative normalization, after the calibration procedure, was checked by comparing the intensities of the mercury pencil lamp lines emitted in the overlap region of the two spectrograph's ranges. A $2 \%$ difference in the measured intensities was found, which was taken as an estimate of the systematic uncertainty on the spectrograph relative calibration procedure.

\section{Measurement of air fluorescence spectrum}

The fluorescence spectrum in air-like mixtures has been measured at the Van de Graaff. Details of the beam and apparatus were given in Section 3.1. Measurements were performed at a gas pressure of $800 \mathrm{hPa}$ and temperature of $293 \mathrm{~K}$. A blow-up of the spectrum in the wavelength region between $343 \mathrm{~nm}$ and $360 \mathrm{~nm}$ is shown in Fig. 3. Notice that the observed lineshapes show an asymmetric tail towards smaller wavelengths, due to the convolution of the rotational levels with the finite resolution of the spectrograph. Thus, in general, the integral counts associated with a given band can have contributions also from nearby bands. Rather than attempting to disentangle individual band integrals, which would result in additional systematic uncertainties, the band intensities were estimated by integrating the CCD counts in a wavelength interval around each band. An example of the procedure is shown in Fig. 3. Wavelength intervals up to several nm were used, depending on the distance of nearby bands and the amount of rotational contribution. In any case, most of the signal of each band is contained in only $2 \mathrm{~nm}$. In the following, the wavelength of the main band is used to identify the corresponding wavelength interval. The measurement of the light intensity in wavelength intervals of $\mathrm{nm}$ size is well suited for the purpose of calibration of the cosmic ray fluorescence detection technique, and assigning the full intensity to the main band of the wavelength interval will result in a negligible systematic uncertainty.

The measured fluorescence spectrum contained a small level of background, 


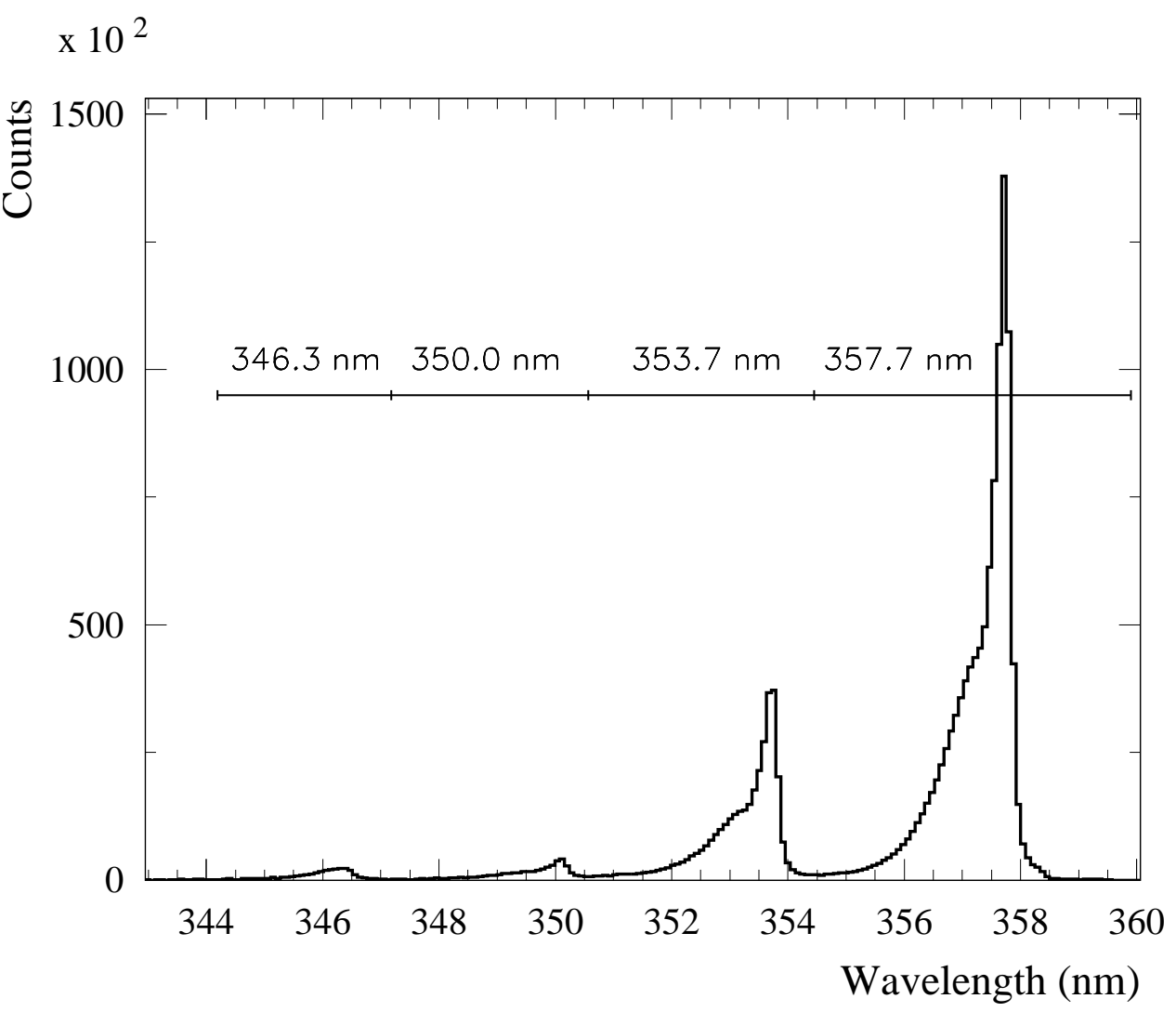

Fig. 3. Example of band integrals. Horizontal lines indicate the wavelength ranges used for the integration of the band fluorescence signal. The main fluorescence band corresponding to each interval is also given.

which was estimated in selected wavelength regions where fluorescence bands were not present. For each of these background regions, the average number of background counts per wavelength bin was determined. In order to estimate the background level under the fluorescence bands, a linear interpolation was performed between the measurements of consecutive background wavelength regions. The procedure gave an estimate of the average number of background counts in each wavelength bin over the full wavelength region. The background level was found to be approximately constant. The amount of background under each fluorescence band was determined by summing the estimated average number of background counts for each bin of the wavelength interval associated to the band. The band intensity was then determined by subtracting the corresponding background.

Several fluorescence bands (346.3, 350.0, 353.7, 357.7, 366.1, and $367.2 \mathrm{~nm}$ ) were common to the $284-369 \mathrm{~nm}$ and the $344-429 \mathrm{~nm}$ spectrograph's ranges. The measured intensity of these bands was used to normalize the two spectrograph's ranges. In fact, any change in the beam intensity or position during the short time ( $30 \mathrm{~s})$ needed by the spectrograph to move from the first to the 
second wavelength range would result in a difference in the measured intensity of the common bands. The intensity of the common bands was found to be $2 \%$ lower in the 344-429 $\mathrm{nm}$ range, and the intensity of all measured bands in this range were corrected correspondingly. The smallness of the correction illustrates the good stability of the beam and the reliability of the relative calibration of the spectrum.

The measured spectrum with the $78 \% \mathrm{~N}_{2}-21 \% \mathrm{O}_{2}-1 \%$ Ar mixture is shown in Fig. 4. The measured intensities, $I_{\lambda}$, relative to the intensity of the $337 \mathrm{~nm}$

\begin{tabular}{|c|c|c||c|c|c|}
\hline$\lambda(\mathrm{nm})$ & $\lambda$ interval $(\mathrm{nm})$ & $I_{\lambda}(\%)$ & $\lambda(\mathrm{nm})$ & $\lambda$ interval $(\mathrm{nm})$ & $I_{\lambda}(\%)$ \\
\hline 296.2 & $292.5-297.0$ & $5.16 \pm 0.29$ & 366.1 & $363.2-366.4$ & $1.13 \pm 0.08$ \\
297.7 & $297.0-299.6$ & $2.77 \pm 0.13$ & 367.2 & $366.4-367.5$ & $0.54 \pm 0.04$ \\
302.0 & $301.5-303.3$ & $0.41 \pm 0.06$ & 371.1 & $367.6-371.7$ & $4.97 \pm 0.22$ \\
308.0 & $306.8-309.3$ & $1.44 \pm 0.10$ & 375.6 & $371.7-376.3$ & $17.87 \pm 0.63$ \\
311.7 & $309.3-312.3$ & $7.24 \pm 0.27$ & 380.5 & $376.3-381.4$ & $27.2 \pm 1.0$ \\
313.6 & $312.3-314.1$ & $11.05 \pm 0.41$ & 385.8 & $383.0-386.0$ & $0.50 \pm 0.08$ \\
315.9 & $314.1-316.7$ & $39.3 \pm 1.4$ & 387.7 & $386.0-388.0$ & $1.17 \pm 0.06$ \\
317.6 & $317.0-318.4$ & $0.46 \pm 0.06$ & 388.5 & $388.0-388.7$ & $0.83 \pm 0.04$ \\
326.8 & $325.6-327.1$ & $0.80 \pm 0.08$ & 391.4 & $388.7-392.1$ & $28.0 \pm 1.0$ \\
328.5 & $327.1-329.0$ & $3.80 \pm 0.14$ & 394.3 & $392.1-394.9$ & $3.36 \pm 0.15$ \\
330.9 & $329.0-331.3$ & $2.15 \pm 0.12$ & 399.8 & $394.9-400.5$ & $8.38 \pm 0.29$ \\
333.9 & $331.3-334.3$ & $4.02 \pm 0.18$ & 405.0 & $400.5-406.6$ & $8.07 \pm 0.29$ \\
337.1 & $334.3-338.4$ & 100.00 & 414.1 & $412.5-414.4$ & $0.49 \pm 0.07$ \\
346.3 & $344.2-347.2$ & $1.74 \pm 0.11$ & 420.0 & $416.6-420.6$ & $1.75 \pm 0.10$ \\
350.0 & $347.2-350.6$ & $2.79 \pm 0.11$ & 423.6 & $420.7-424.0$ & $1.04 \pm 0.11$ \\
353.7 & $350.6-354.4$ & $21.35 \pm 0.76$ & 427.0 & $424.0-427.4$ & $7.08 \pm 0.28$ \\
357.7 & $354.4-359.9$ & $67.4 \pm 2.4$ & 427.8 & $427.4-428.6$ & $4.94 \pm 0.19$ \\
\hline
\end{tabular}

Table 1

Measured fluorescence band intensities in dry air at $800 \mathrm{hPa}$ pressure and $293 \mathrm{~K}$ temperature. The intensity of the $337 \mathrm{~nm}$ band was used for normalization. The wavelength intervals used for the signal integration are also reported.

band, of 34 fluorescence bands are reported in Table 1, together with the corresponding wavelength intervals used for the signal integration. The fluorescence spectrum was measured ten times, and from the observed r.m.s. of the ten measurements of each band intensity, uncertainties were estimated. To obtain the total uncertainty quoted in Table 1, a systematic uncertainty 


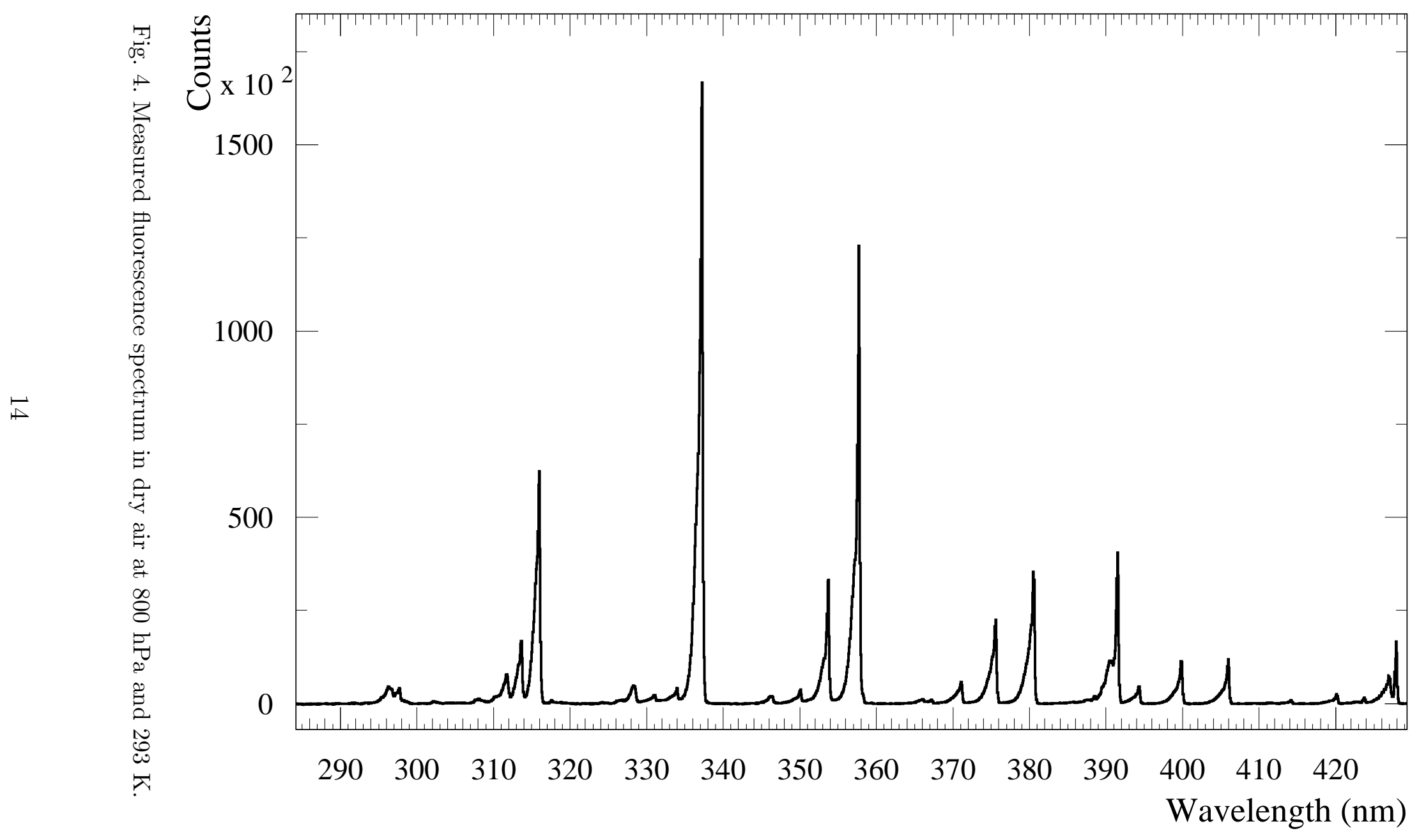


of $3.5 \%$, assigned to the calibration of the relative spectrograph sensitivity, was summed in quadrature. The systematic uncertainty of the relative spectrograph sensitivity accounts for the uncertainty on the absolute calibration of the lamp emission (2.5\%), the difference in the mercury pencil lamp line intensities measured in the two wavelength ranges of the spectrograph $(2 \%)$, and the uncertainty associated with the spatial distribution of the fluorescence light source (1.5\%). The fluorescence light is in fact emitted along the beam axis, and the calibration procedure of relative spectrograph sensitivity (cf. Section 3.4) derived calibration factors from measurements with the calibration lamp placed in different positions along the beam axis. If calibration factors obtained only from a measurement with the calibration lamp placed at the optics center are used, the relative fluorescence band intensities changed by at most 3\%. Half of this shift was conservatively taken as an estimate of the associated systematic uncertainty.

Several checks were performed. The linearity of the fluorescence emission with beam currents from 0.2 to $14 \mu \mathrm{A}$ was verified. Possible systematic effects due to the beam position were investigated. The fluorescence spectrum was measured with the beam moved $\pm 1 \mathrm{~cm}$ in the directions transverse to the nominal beam axis. No difference beyond the statistical uncertainty in the relative intensities of the fluorescence bands was found. Several models for background evaluation were tested, which always resulted in changes of the relative band intensities within the quoted uncertainties. A measurement of the fluorescence spectrum in pure nitrogen gas was performed, which showed that all the observed bands in the air fluorescence spectrum are associated with nitrogen excitation.

In order to assess the relevance of argon on air fluorescence, the fluorescence spectrum emitted by a $79 \% \mathrm{~N}_{2}-21 \% \mathrm{O}_{2}$ gas mixture was measured at the same pressure and temperature conditions as the mixture with argon. Fig. 5 shows the correlation of the relative intensities of the 34 fluorescence bands measured with the $79 \% \mathrm{~N}_{2}-21 \% \mathrm{O}_{2}$ and the $78 \% \mathrm{~N}_{2}-21 \% \mathrm{O}_{2}-1 \%$ Ar mixture. A linear fit yielded an intercept consistent with zero and a slope consistent with unity, within a few per mil, which indicates that the two spectra are compatible to a high degree of accuracy. On the basis of this observation, the effect of argon on air fluorescence at atmospheric pressure is completely negligible.

The $2 \mathrm{P}$ and $1 \mathrm{~N}$ bands of nitrogen emission can be easily identified in our measured fluorescence spectrum. A group of weak bands (302.0, 308.0, 317.6, $346.3,366.1,387.7 \mathrm{~nm}$ ) is consistent with the Gaydon-Herman (GH) bands [16] [29]. The $\mathrm{GH}(0,1-3)$ bands at 296.7, 311.9 and $328.3 \mathrm{~nm}$ are also present, with a large overlap with the $2 \mathrm{P}(3,1-3)$ bands at $296.2,311.7$ and $328.5 \mathrm{~nm}$. The excitation of the Gaydon-Herman bands by electrons in air at atmospheric pressure has already been observed [30]. Within a vibrational band system, the relative intensities are expected to be equal to the ratio of the corresponding Einstein coefficients, and can be calculated [31] [32]. However, 


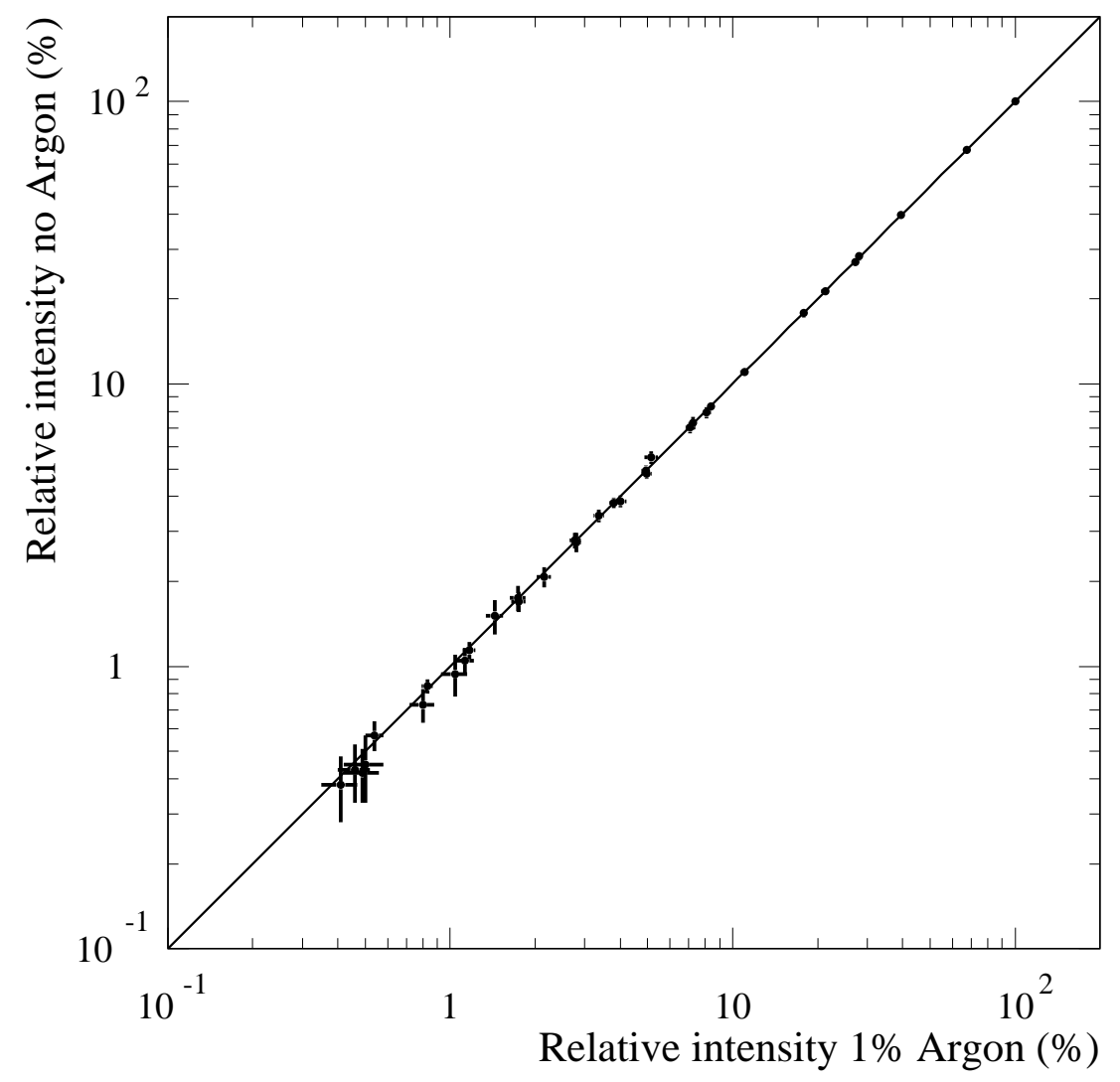

Fig. 5. Effect of argon on the air fluorescence yield. Relative intensities of the 34 fluorescence bands measured in a $79 \% \mathrm{~N}_{2}-21 \% \mathrm{O}_{2}$ mixture vs the corresponding intensities measured in the $78 \% \mathrm{~N}_{2}-21 \% \mathrm{O}_{2}-1 \%$ Ar mixture. A linear fit of the data is also shown, which indicates that the yield in the two gas mixtures is the same to a high level of accuracy.

the intensities reported in Table 1 cannot be directly compared with the theoretical expectations, due to the contamination from nearby bands. A simple extrapolation of the band's spectral shape was used to estimate the contamination, and intensities were corrected accordingly. The corresponding relative intensities for the $2 \mathrm{P}(0, \mathrm{i}), 2 \mathrm{P}(1, \mathrm{i}), 2 \mathrm{P}(2, \mathrm{i})$, and $1 \mathrm{~N}(0, \mathrm{i})$ bands are reported in Table 2, together with the theoretical predictions [31] [32]. Only bands where the contamination could be reliably estimated are included in Table 2. The measured relative intensities are close to the theoretical expectations and this represents an independent cross-check of the relative spectrograph sensitivity calibration. 


\begin{tabular}{|c|c|c|c|c|c|c|c|}
\hline Band & $\begin{array}{c}\lambda \\
(\mathrm{nm})\end{array}$ & $\begin{array}{c}\text { Measured } \\
(\%)\end{array}$ & $\begin{array}{c}\text { Theory } \\
(\%)\end{array}$ & Band & $\begin{array}{c}\lambda \\
(\mathrm{nm})\end{array}$ & $\begin{array}{c}\text { Measured } \\
(\%)\end{array}$ & $\begin{array}{c}\text { Theory } \\
(\%)\end{array}$ \\
\hline $2 \mathrm{P}(0,0)$ & 337.1 & 100. & 100. & $2 \mathrm{P}(2,1)$ & 313.6 & 100. & 100. \\
\hline $2 \mathrm{P}(0,1)$ & 357.7 & 67.4 & 67.5 & $2 \mathrm{P}(2,2)$ & 330.9 & 7.9 & 7.3 \\
\hline $2 \mathrm{P}(0,2)$ & 380.5 & 26.5 & 27.2 & $2 \mathrm{P}(2,3)$ & 350.0 & 16.1 & 16.9 \\
\hline $2 \mathrm{P}(0,3)$ & 405.0 & 7.9 & 8.4 & $2 \mathrm{P}(2,4)$ & 371.1 & 38.7 & 40.0 \\
\hline $2 \mathrm{P}(1,0)$ & 315.9 & 100. & 100. & $2 \mathrm{P}(2,5)$ & 394.3 & 29.6 & 31.1 \\
\hline $2 \mathrm{P}(1,1)$ & 333.9 & 4.0 & 4.9 & $2 \mathrm{P}(2,6)$ & 420.0 & 15.5 & 15.5 \\
\hline $2 \mathrm{P}(1,2)$ & 353.7 & 47.8 & 46.6 & $1 \mathrm{~N}(0,0)$ & 391.4 & 100. & 100 \\
\hline $2 \mathrm{P}(1,3)$ & 375.6 & 41.9 & 41.4 & $1 \mathrm{~N}(0,1)$ & 427.8 & 31.8 & 32.5 \\
\hline $2 \mathrm{P}(1,4)$ & 399.8 & 20.8 & 20.4 & & & & \\
\hline $2 \mathrm{P}(1,5)$ & 427.0 & 9.9 & 7.5 & & & & \\
\hline
\end{tabular}

Table 2

Measured relative intensities within bands and theoretical expectations based on the ratio of Einstein coefficients.

\section{$5 \quad$ Pressure dependence of the $337 \mathrm{~nm}$ band}

A precise measurement of the pressure dependence of the $337 \mathrm{~nm}$ band has been performed at the AWA facility. The experimental set-up has been described in Section 3.2. A narrow band interference filter centered at $337 \mathrm{~nm}$ was used for this measurement. The filter transmission curve is shown in Fig. 6. together with the fluorescence emission bands as measured by the spectrograph (Section 4). The filter transmission was measured by three different groups of the AIRFLY collaboration, which yielded consistent results at the percent level. The filter selects mainly the $337 \mathrm{~nm}$ band, the contamination from neighbouring bands being only $1.7 \%$.

In order to check linearity and possible pedestal changes within a run, the electron beam was operated in a mode allowing the bunch charge to fluctuate over a wide range. An example of correlation of the photomultiplier signal $S_{P M T}$ and the ICT signal $S_{I C T}$ is shown in Fig. 7, for a run at $180 \mathrm{hPa}$ in pure nitrogen. A linear fit $S_{P M T}=S_{F L} \cdot S_{I C T}+b$ to the data was performed, and the fitted slope $S_{F L}$ was taken as an estimator of the fluorescence signal. The same procedure, applied on data taken with the shutter closed in front of the photomultiplier tube, allowed an estimate of the background level. A typical background correlation to the beam intensity is also shown in Fig. 7. Several background runs were taken during the pressure scans. In addition, during the fluorescence runs, the background was monitored by a second photomultiplier, 


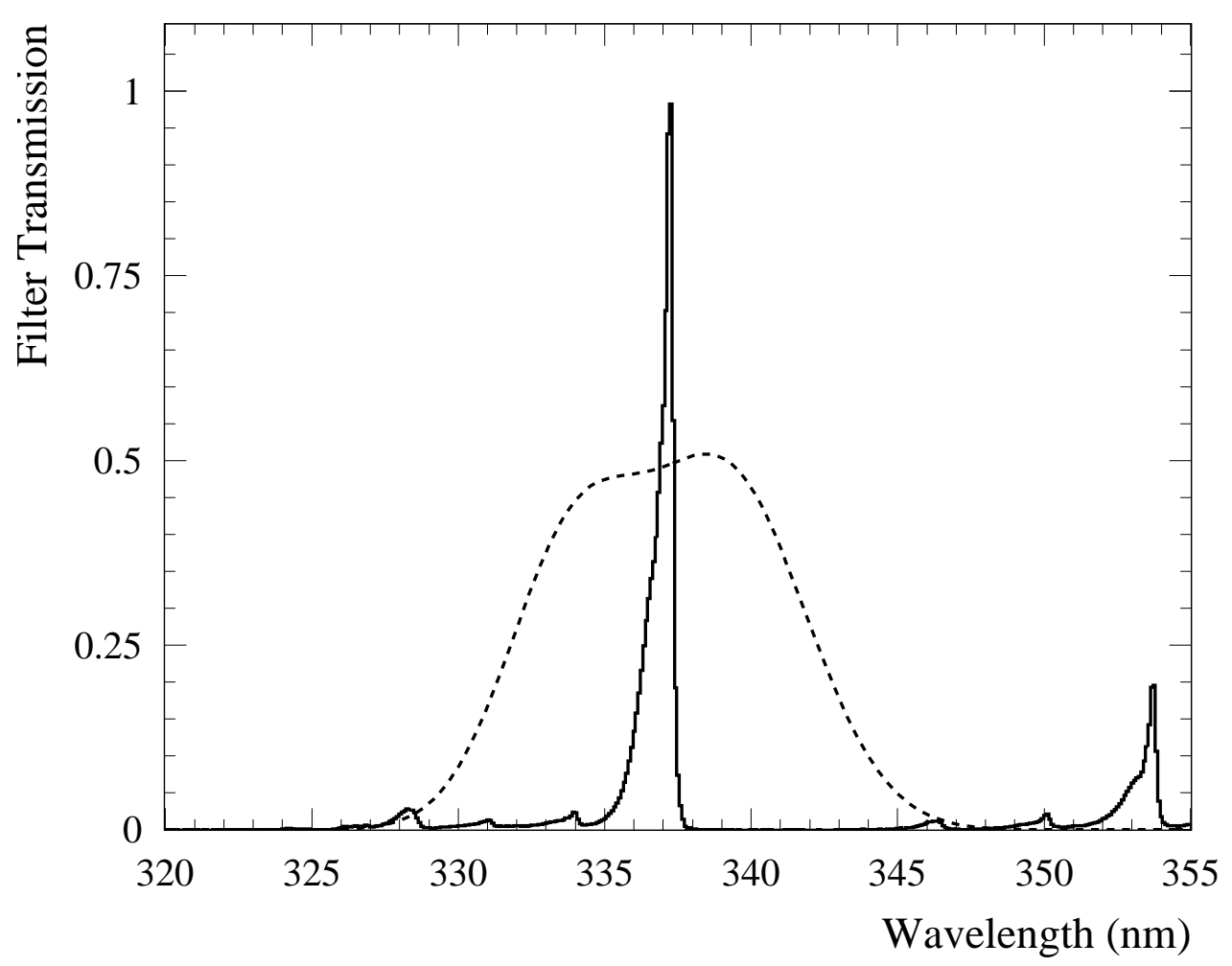

Fig. 6. Transmission of the interference filter used to select the $337 \mathrm{~nm}$ band (dashed line). The measured fluorescence spectrum in arbitrary units is shown as a full line.

and found to be stable within its statistical uncertainty. The background slope was subtracted from the signal slope, and in the following $S_{F L}$ will refer to the background subtracted signal.

Measurements were performed with dry air-like mixtures and pure nitrogen. The gas pressure in the fluorescence chamber was varied in the range from 2 to $1000 \mathrm{hPa}$, and for each pressure $p$ a value $S_{F L}(p)$ was measured. The gas temperature was $293 \mathrm{~K}$. Only a couple of minutes for each pressure point was necessary to collect enough statistics, thus minimizing the possibility of systematic changes of the beam and the gas condition, as well as the detector response.

The measured fluorescence signal $S_{F L}^{g a s}(p)$ in nitrogen or air is proportional to (cf. Eq. (8)):

$$
S_{F L}^{g a s}(p) \propto N_{337}^{g a s}=\left(\frac{d E}{d X}\right)_{g a s} \frac{p}{R_{g a s} T} D_{g a s}(p) \frac{Y_{\mathrm{N}_{2}}^{0}(337) f_{\mathrm{N}_{2}}}{1+\frac{p}{p_{g a s}^{\prime}(337)}},
$$




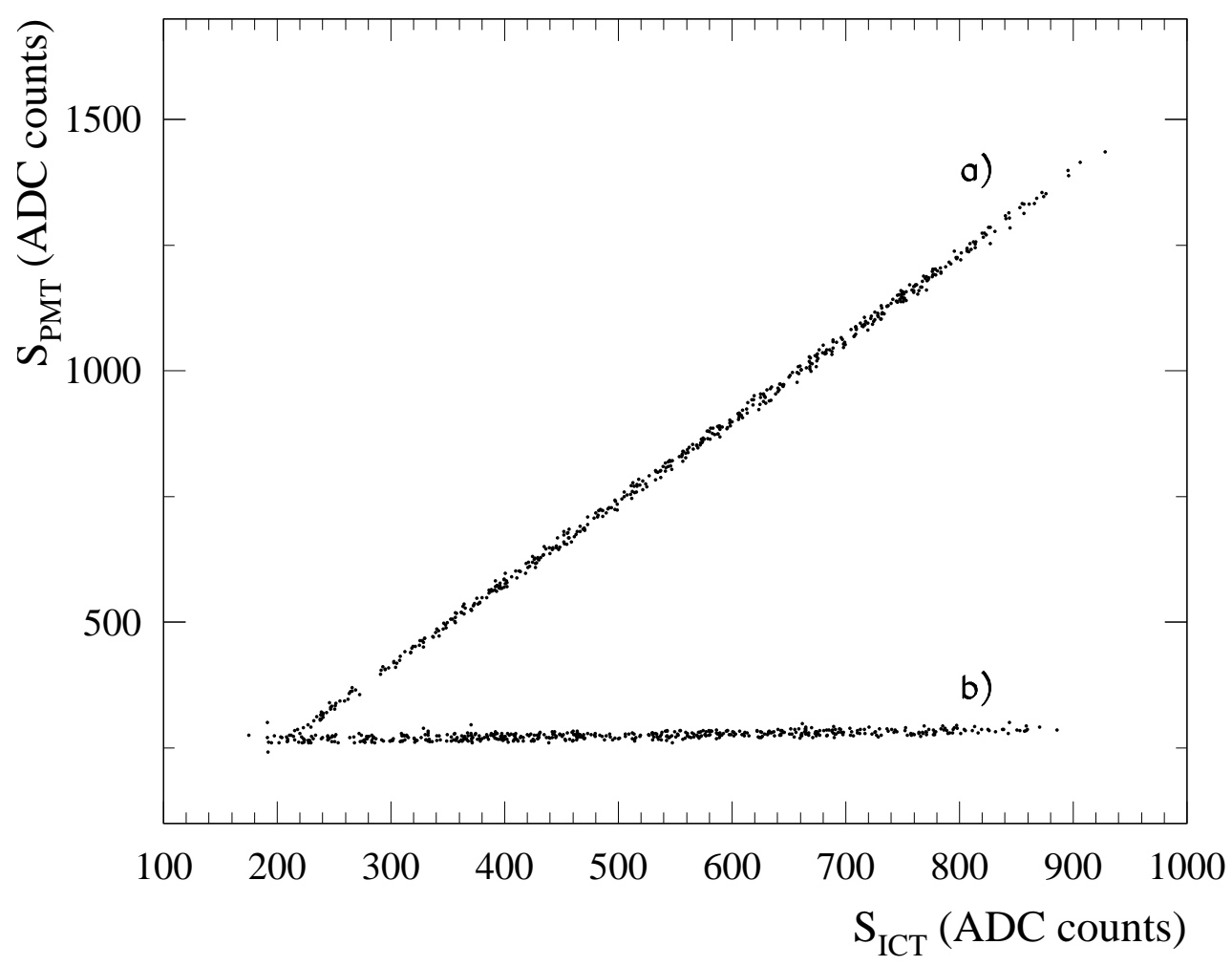

Fig. 7. Correlation between the photomultiplier signal and the beam ICT signal: a) fluorescence run in pure nitrogen at $180 \mathrm{hPa}$, b) background run.

where $f_{\mathrm{N}_{2}}=1$ for pure nitrogen. Notice that the fluorescence emission is essentially due to excitation by secondary electrons produced in the gas by the beam electrons [33] [34]. As the gas pressure goes down, an increasing fraction of secondary electrons does not stop in the photomultiplier field of view, and correspondingly part of the fluorescence emission is not detected. Neglecting the losses due to the secondary electrons escaping the field of view would cause an overestimation of the quenching reference pressure $p^{\prime}$ [34].

In order to avoid this bias, we have studied the ratio of the $337 \mathrm{~nm}$ fluorescence signal in pure nitrogen to the signal in air, $r_{\mathrm{N}_{2}}=S_{F L}^{\mathrm{N}_{2}}(p) / S_{F L}^{a i r}(p)$. The effective length $D(p)$ is expected to cancel in the ratio, since secondary electron interactions are similar in nitrogen and air thanks to their close molecular masses. This was verified with a detailed GEANT4 [36] simulation of the experiment. The pressure dependence of the fluorescence signal ratio thus becomes:

$$
r_{\mathrm{N}_{2}}=\frac{\left(\frac{d E}{d X}\right)_{\mathrm{N}_{2}} / R_{\mathrm{N}_{2}}}{\left(\frac{d E}{d X}\right)_{\text {air }} f_{\mathrm{N}_{2}} / R_{\text {air }}} \cdot \frac{1+p\left(\frac{f_{\mathrm{N}_{2}}}{p_{\mathrm{N}_{2}}(337)}+\frac{f_{\mathrm{O}_{2}}}{p_{\mathrm{O}_{2}}^{\prime}(337)}\right)}{1+\frac{p}{p_{\mathrm{N}_{2}}^{\prime}(337)}}
$$

where $f_{\mathrm{N}_{2}}$ and $f_{\mathrm{O}_{2}}$ are the fraction of nitrogen and oxygen molecules in the air 


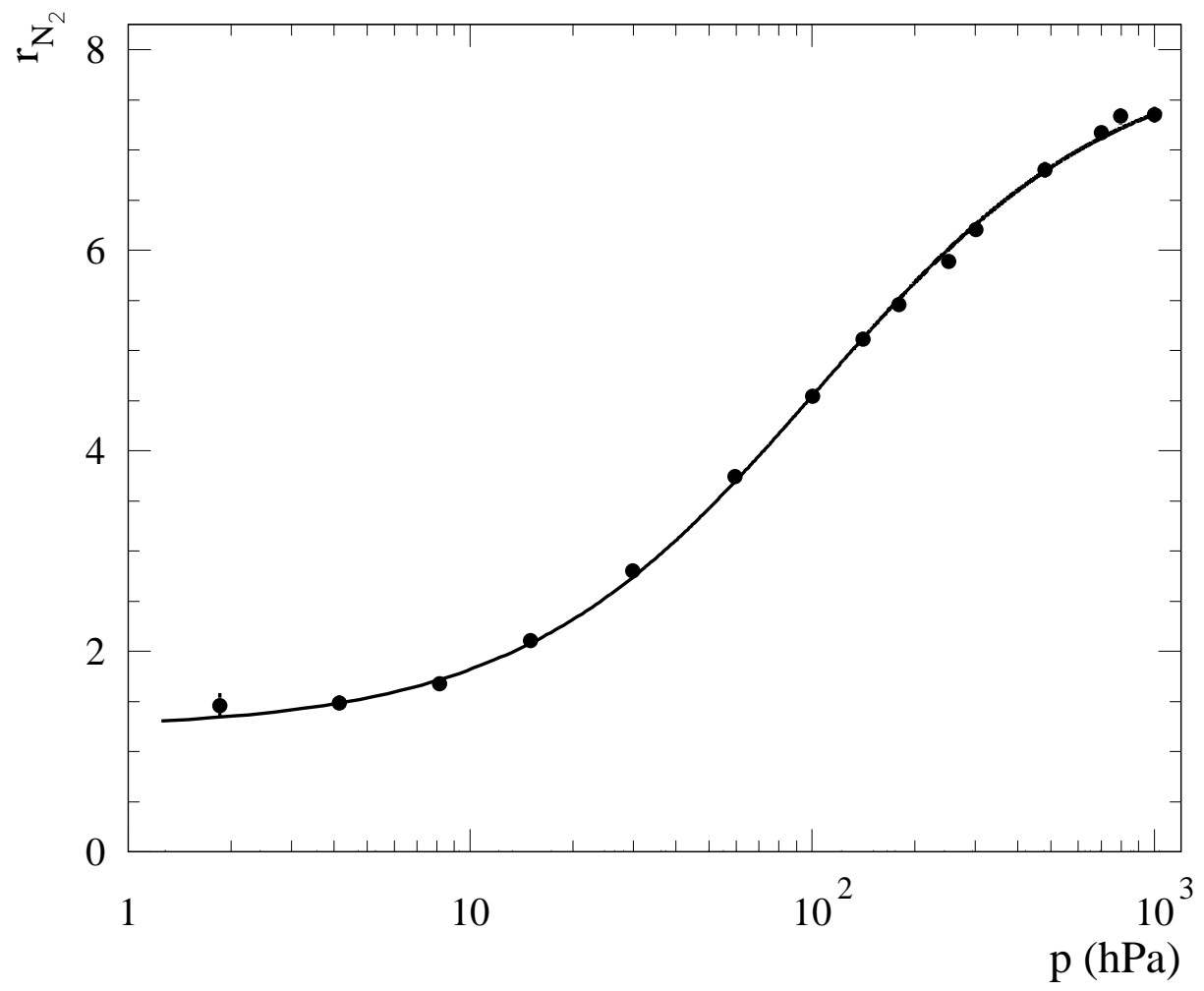

Fig. 8. Pressure dependence of the ratio of the $337 \mathrm{~nm}$ band signal in nitrogen to the signal in dry air. The full line is the result of a fit to the full pressure range described in the text.

mixture. The collisional energy loss $(d E / d X)$ in nitrogen and air at $14 \mathrm{MeV}$ was calculated with the method described in [35].

The measured pressure dependence of the ratio $r_{\mathrm{N}_{2}}$ of the $337 \mathrm{~nm}$ band is shown in Fig. 8, The $78 \% \mathrm{~N}_{2}-21 \% \mathrm{O}_{2}-1 \%$ Ar air mixture was used for this measurement. The full line is the result of the best fit of Eq. (10) to the data in the range 2-1000 $\mathrm{hPa}$, with $p_{\mathrm{N}_{2}}^{\prime}$ and $p_{\mathrm{O}_{2}}^{\prime}$ as free parameters. The best fit yielded:

$$
\begin{aligned}
& p_{\mathrm{N}_{2}}^{\prime}(337)=103.7 \pm 2.7 \mathrm{hPa}, \\
& p_{\mathrm{O}_{2}}^{\prime}(337)=3.796 \pm 0.076 \mathrm{hPa},
\end{aligned}
$$

where the quoted uncertainties are statistical only, and a $\chi^{2} /$ n.d.f. $=0.9$.

From Eq. (5), a value of $p_{\text {air }}^{\prime}$ for the $78 \% \mathrm{~N}_{2}-21 \% \mathrm{O}_{2}-1 \%$ Ar mixture was 


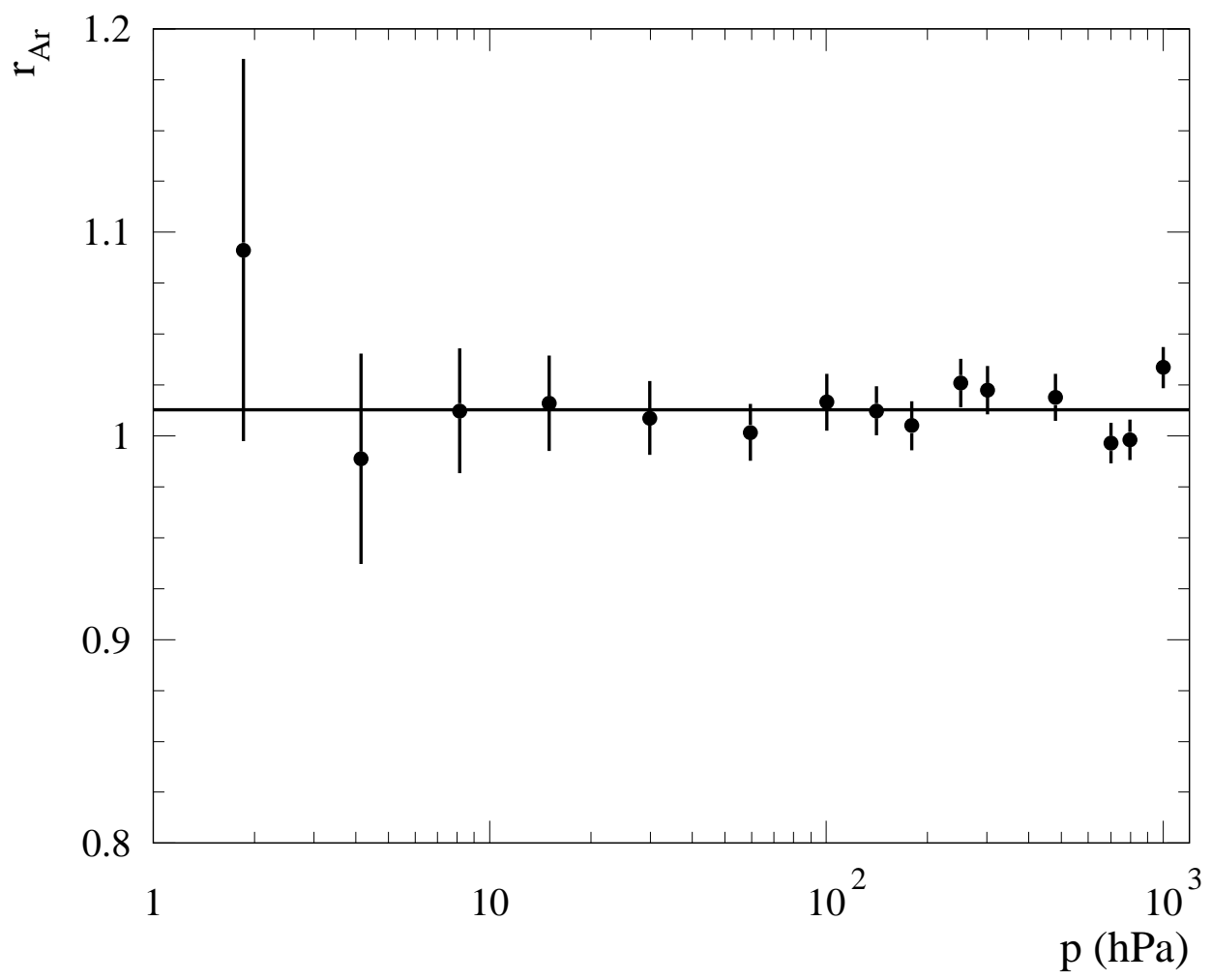

Fig. 9. Ratio of the fluorescence signal for air-like mixture with $1 \%$ Ar to the mixture without $\mathrm{Ar}$ as a function of pressure.

estimated:

$$
p_{\text {air }}^{\prime}(337)=15.89 \pm 0.33 \mathrm{hPa},
$$

where the statistical uncertainty was calculated from the covariance matrix of the fitted $p_{\mathrm{N}_{2}}^{\prime}$ and $p_{\mathrm{O}_{2}}^{\prime}$ parameters.

Notice that the contribution of argon to the quenching of the $337 \mathrm{~nm}$ band was neglected in Eq. (10). This is justified by the comparison of the pressure scan measurements performed with two air-like mixtures. The ratio $r_{\mathrm{Ar}}$ of the fluorescence signal measured with the $78 \% \mathrm{~N}_{2}-21 \% \mathrm{O}_{2}-1 \%$ Ar mixture to the one with the $79 \% \mathrm{~N}_{2}-21 \% \mathrm{O}_{2}$ mixture as a function of pressure is shown in Fig. 9. A linear fit to the data yielded an intercept of $1.011 \pm 0.006$ and a slope consistent with zero at the level of $10^{-5}$, with a $\chi^{2} /$ n.d.f. $=1.0$. The effect of argon to the quenching of the $337 \mathrm{~nm}$ band is thus negligible at all pressures.

Several checks were performed in order to estimate the systematic uncertainties on the measured $p^{\prime}$. The stability of the photomultiplier gain was moni- 
tored during the measurements by a blue LED. The PMT gain was found to be stable at the $1 \%$ level, with negligible effect on the $p^{\prime}$ determination. The range used to fit the fluorescence signal $S_{P M T}$ as a function of the ICT signal $S_{I C T}$ was varied over a wide interval. The lower limit in the fit of the pressure dependence was changed from $2 \mathrm{hPa}$ to $30 \mathrm{hPa}$. The background was changed between its minimum and maximum measured value. The observed shifts in the $p^{\prime}$ value due to these variations were taken as estimates of the systematic uncertainties. A systematic uncertainty of $0.1 \mathrm{hPa}$ was assigned from the uncertainty in the measurement of the absolute pressure. The different contributions to the systematic uncertainty, together with their quadrature sum, are summarized in Table 3. The total uncertainty on the measured $p^{\prime}$, given by the quadrature sum of the statistical and systematic uncertainties, is also quoted.

\begin{tabular}{|c|c|c|c|}
\hline Source & $\Delta p_{\mathrm{N}_{2}}^{\prime}(\mathrm{hPa})$ & $\Delta p_{\mathrm{O}_{2}}^{\prime}(\mathrm{hPa})$ & $\Delta p_{\text {air }}^{\prime}(\mathrm{hPa})$ \\
\hline slope fit range & 3.6 & 0.10 & 0.50 \\
\hline background & 2.1 & 0.09 & 0.40 \\
\hline pressure fit range & 0.3 & 0.01 & 0.03 \\
\hline absolute pressure & 0.1 & 0.10 & 0.10 \\
\hline TOTAL SYST. & 4.2 & 0.17 & 0.65 \\
\hline \hline TOTAL UNCERTAINTY & 5.0 & 0.19 & 0.73 \\
\hline
\end{tabular}

Table 3

Estimated systematic uncertainties on the quenching reference pressure of the 337 nm fluorescence band. The total uncertainty, given by the the quadrature sum of statistical and systematic uncertainties, is also given in the last line.

The method of the nitrogen to air ratio has the advantage of eliminating the bias due to secondary electrons escaping the field of view of the photomultiplier. On the other hand, a direct interpretation of the data with Eq. (9) allows a valuable check of our understanding of the experiment. For this purpose a detailed GEANT4 [36] simulation of the experiment was performed. The PENELOPE [37] package in GEANT4 was used, which allows the tracking of secondary electrons down to $250 \mathrm{eV}$. In the simulation, the fluorescence emission was assumed to be proportional to the energy deposited by the particles in the gas. The effective length, which takes into account the field of view losses, was found to be well parameterized by $D(p)=D_{1000} F(p)$ from the Monte Carlo simulations, where $D_{1000}$ is the effective length at $1000 \mathrm{hPa}$ and $F(p)=(p / 1000)^{0.027}$. The pressure dependence of the fluorescence signal can thus be fitted with the function:

$$
S_{F L}^{g a s}(p)=C \frac{p}{1+\frac{p}{p_{g a s}^{\prime}(337)}} F(p),
$$


leaving $C$ and $p_{\text {gas }}^{\prime}$ as free parameters. A direct fit of the pressure dependence of the $337 \mathrm{~nm}$ band fluorescence signal $S_{F L}^{\mathrm{N}_{2}}$ in nitrogen with Eq. (14) yielded:

$$
p_{\mathrm{N}_{2}}^{\prime}(337)=101.0 \pm 0.6 \mathrm{hPa},
$$

while a fit of the pressure dependence of the $337 \mathrm{~nm}$ fluorescence signal $S_{F L}^{\text {air }}$ in the $78 \% \mathrm{~N}_{2}-21 \% \mathrm{O}_{2}-1 \%$ Ar mixture gave:

$$
p_{\text {air }}^{\prime}(337)=15.34 \pm 0.53 \mathrm{hPa},
$$

in good agreement with (11) and (13). To avoid the difficulties of simulations at very low gas densities, we limited the fit to a minimum pressure of 15 $\mathrm{hPa}$. The fact that two different ways of taking into account the effect of secondary electrons escaping the field of view, either by cancelation in the nitrogen to air fluorescence ratio or by a parameterization of the light losses with simulations, yielded consistent values of $p^{\prime}$ strengthen the confidence in our quenching reference pressure measurements. Also, this consistency shows that the simple ansatz of Eqs. (4)-(5) does indeed provide a good description of our data.

It should be stressed that the effect of secondary electrons escaping the detector field of view cannot be neglected in the measurement of $p^{\prime}$. In fact, a fit of the data with $F(p)=1$ in Eq. (14) gave $p_{\mathrm{N}_{2}}^{\prime}(337)=115.5 \mathrm{hPa}$ and $p_{\text {air }}^{\prime}(337)=19.95 \mathrm{hPa}$, showing a significant bias towards larger $p^{\prime}$ values.

\section{Pressure dependence of the spectrum bands}

The pressure dependence of the air fluorescence spectrum bands has been measured at the Van de Graaff. The experimental arrangement and beam conditions have been described in Sections 3 and 4 . The air fluorescence spectrum was measured for gas pressures in the range from 4 to $1000 \mathrm{hPa}$, at temperature $293 \mathrm{~K}$. For each pressure, the spectrum band intensities relative to the $337 \mathrm{~nm}$ band were measured following the procedure described in Section 4. From Eq. (9), the pressure dependence of the relative intensity of a band of wavelength $\lambda$ is given by:

$$
I_{\lambda}(p)=C \frac{1+\frac{p}{p_{a i r}^{\prime}(337)}}{1+\frac{p}{p_{a i r}^{\prime}(\lambda)}} .
$$

The measured $I_{\lambda}(p)$ are shown in Figs. 10, 12 for one of the ten pressure scans which were performed. We could not measure the pressure dependence for 

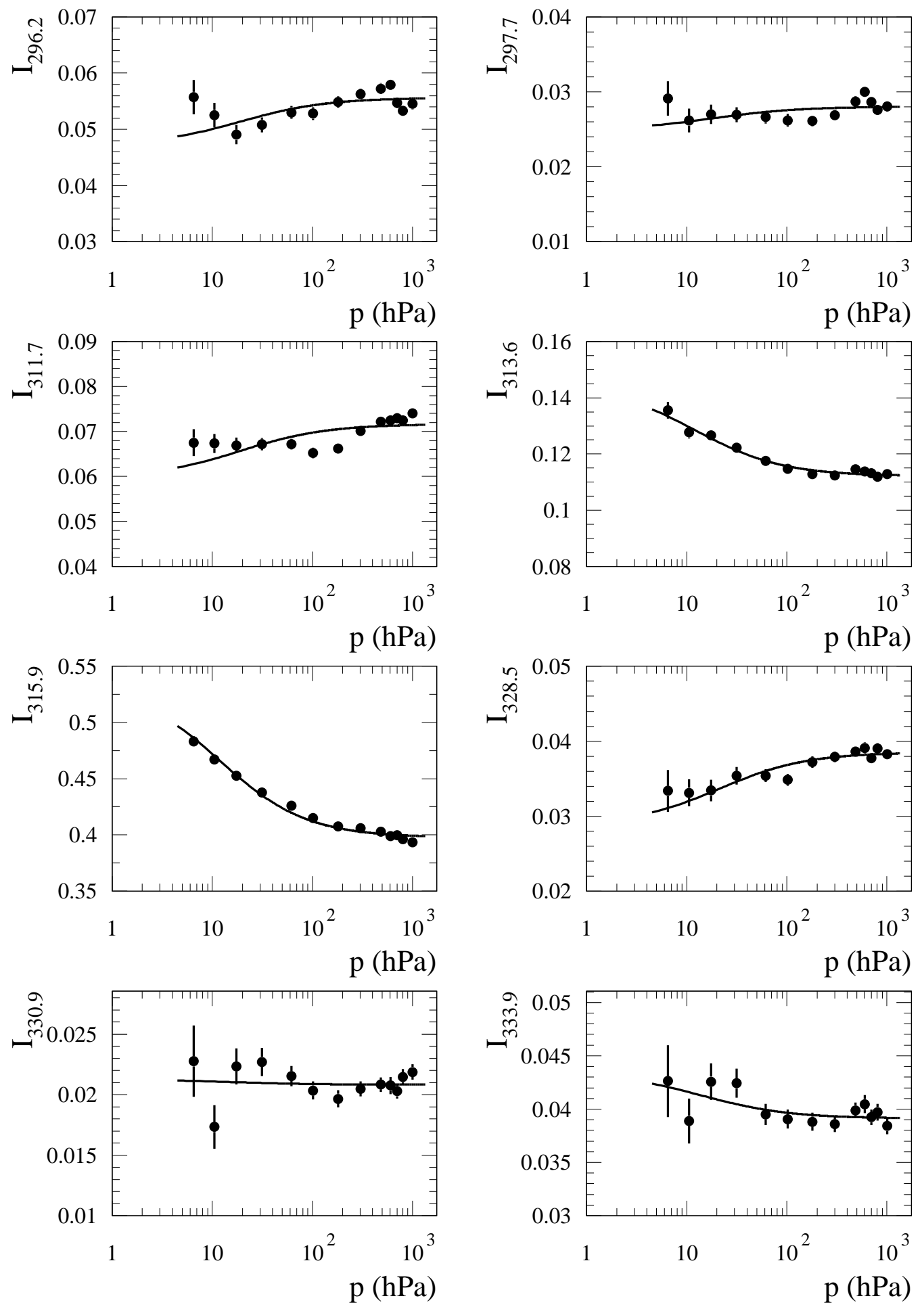

Fig. 10. Pressure dependence of the air fluorescence bands shown in increasing order from $296.2 \mathrm{~nm}$ to $333.9 \mathrm{~nm}$. Intensities are relative to the $337 \mathrm{~nm}$ band. Errors are statistical only. The full line is the result of a fit described in the text. 

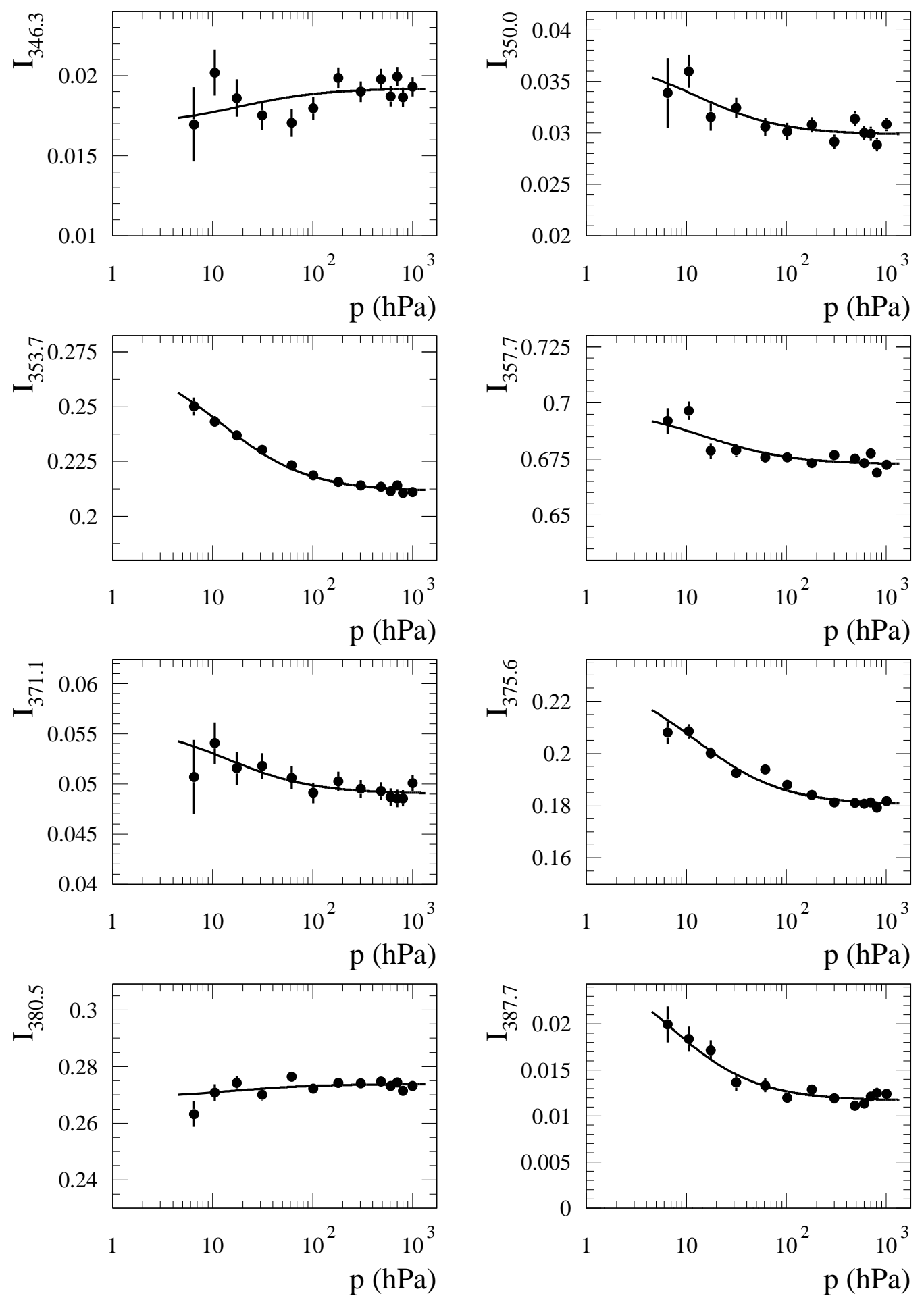

Fig. 11. Pressure dependence of the air fluorescence bands shown in increasing order from $346.3 \mathrm{~nm}$ to $387.7 \mathrm{~nm}$. Intensities are relative to the $337 \mathrm{~nm}$ band. Errors are statistical only. The full line is the result of a fit described in the text. 

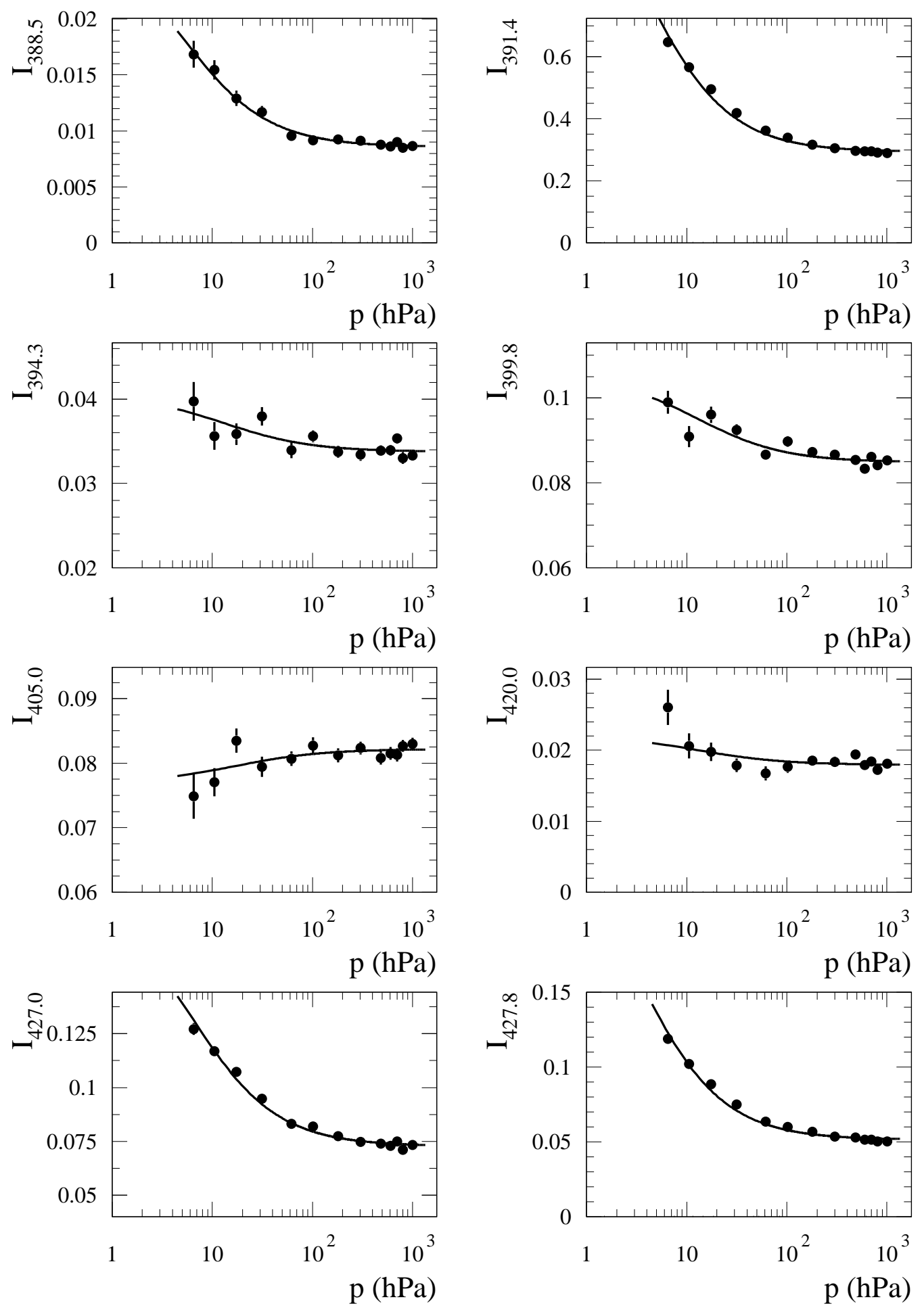

Fig. 12. Pressure dependence of the air fluorescence bands shown in increasing order from $388.5 \mathrm{~nm}$ to $427.8 \mathrm{~nm}$. Intensities are relative to the $337 \mathrm{~nm}$ band. Errors are statistical only. The full line is the result of a fit described in the text. 
nine of the bands in Table 1, due to their low intensity. The full line is the result of the best fit of Eq. (17) to the data, leaving $C$ and $p_{\text {air }}^{\prime}(\lambda)$ as free parameters. The $337 \mathrm{~nm}$ quenching reference pressure $p_{\text {air }}^{\prime}(337)$ was fixed to the value of $15.89 \mathrm{hPa}$ obtained in Section 5 (Eq. (13)). In principle, the measured $I_{\lambda}(800 \mathrm{hPa})$ given in Table 1 could be used as a constraint for the $C$ parameter, therefore reducing the error on $p_{\text {air }}^{\prime}(\lambda)$. On the other hand, the measured values of $p_{\text {air }}^{\prime}(\lambda)$ and $I_{\lambda}(800 \mathrm{hPa})$ would become correlated. Leaving $C$ free in the fit also minimizes systematic uncertainties, since the calibration of the relative spectrograph sensitivity does not affect in this case the measured quenching reference pressure.

Notice that Eq. (17) describes a model where the pressure dependence of a spectrum band intensity is given by only one quenching reference pressure.This model may not be true in general because more than one band could be contained in the wavelength interval used for the signal integration. From the quality of the fits, however, it appears that the assumption of a single reference pressure is a good approximation. The largest deviation from the model, $<10 \%$, is found for the $311.7 \mathrm{~nm}$ band, which is expected to have contributions of similar size from the $\mathrm{GH}(0,2)$ and $2 \mathrm{P}(3,2)$ bands. A more elaborated model of pressure dependence of the spectrum bands could be used, but since it would not change significantly the application of our measurements to the fluorescence detection of ultra high energy cosmic rays, the simplest approach of Eq. (17) was adopted.

In order to study possible systematic effects, the measurement was repeated several times. Measurements were performed in two separate test beam periods, under different beam conditions and light collection optics. Both the $78 \%$ $\mathrm{N}_{2}-21 \% \mathrm{O}_{2}-1 \%$ Ar and the $79 \% \mathrm{~N}_{2}-21 \% \mathrm{O}_{2}$ air-like mixtures were used. All measurements were found to be compatible within the quoted uncertainties.

The measured $p_{\text {air }}^{\prime}(\lambda)$, obtained from the weighted average of ten measurements, are reported in Table 4. Two uncertainties are quoted: the first is the r.m.s. of the ten measurements, and the second comes from changing the value of $p_{\text {air }}^{\prime}(337)$ in the fit by its total uncertainty $\Delta p_{\text {air }}^{\prime}(337)= \pm 0.73 \mathrm{hPa}$ (see Table 3 ).

Spectrum bands within a system should have the same quenching reference pressure. This may not be true in our measurements, since for each band the signal is integrated over a wavelength interval, which can contain also other bands. However, most of the bands are well separated, and contamination is in general small. Indeed, we observe that most of the bands of a given system have the same $p^{\prime}$ within errors. Bands which deviated from this behavior, like the 387.7 and $427.0 \mathrm{~nm}$ bands, are significantly contaminated by nearby bands. This good agreement gives further confidence on the quality of our pressure dependence measurements. 


\begin{tabular}{|c|c|c||c|c|c|}
\hline Band & $\lambda(\mathrm{nm})$ & $p_{\text {air }}^{\prime}(\lambda)(\mathrm{hPa})$ & Band & $\lambda(\mathrm{nm})$ & $p_{\text {air }}^{\prime}(\lambda)(\mathrm{hPa})$ \\
\hline $2 \mathrm{P}(0,0)$ & 337.1 & $15.89 \pm 0.73$ & $2 \mathrm{P}(2,0)$ & 297.7 & $17.3 \pm 4.0 \pm 0.8$ \\
$2 \mathrm{P}(0,1)$ & 357.7 & $15.39 \pm 0.25 \pm 0.72$ & $2 \mathrm{P}(2,1)$ & 313.6 & $12.27 \pm 0.78 \pm 0.64$ \\
$2 \mathrm{P}(0,2)$ & 380.5 & $16.51 \pm 0.48 \pm 0.72$ & $2 \mathrm{P}(2,2)$ & 330.9 & $16.9 \pm 3.5 \pm 0.76$ \\
$2 \mathrm{P}(0,3)$ & 405.0 & $17.8 \pm 1.5 \pm 0.8$ & $2 \mathrm{P}(2,3)$ & 350.0 & $15.2 \pm 3.7 \pm 0.7$ \\
\hline $2 \mathrm{P}(1,0)$ & 315.9 & $11.88 \pm 0.31 \pm 0.62$ & $2 \mathrm{P}(2,4)$ & 371.1 & $14.8 \pm 1.9 \pm 0.7$ \\
$2 \mathrm{P}(1,1)$ & 333.9 & $15.5 \pm 1.5 \pm 0.7$ & $2 \mathrm{P}(2,5)$ & 394.3 & $13.7 \pm 3.3 \pm 0.7$ \\
$2 \mathrm{P}(1,2)$ & 353.7 & $12.70 \pm 0.34 \pm 0.64$ & $2 \mathrm{P}(2,6)$ & 420.0 & $13.8 \pm 4.0 \pm 0.7$ \\
$2 \mathrm{P}(1,3)$ & 375.6 & $12.82 \pm 0.45 \pm 0.62$ & $2 \mathrm{P}(3,1)$ & 296.2 & $18.5 \pm 5.0 \pm 0.8$ \\
$2 \mathrm{P}(1,4)$ & 399.8 & $13.6 \pm 1.1 \pm 0.6$ & $2 \mathrm{P}(3,2)$ & 311.7 & $18.7 \pm 3.8 \pm 0.8$ \\
$2 \mathrm{P}(1,5)$ & 427.0 & $6.38 \pm 0.68 \pm 0.43$ & $2 \mathrm{P}(3,3)$ & 328.5 & $20.7 \pm 2.6 \pm 0.8$ \\
\hline $1 \mathrm{~N}(0,0)$ & 391.4 & $2.94 \pm 0.58 \pm 0.31$ & $\mathrm{GH}(0,4)$ & 346.3 & $21 \pm 10 \pm 1$ \\
$1 \mathrm{~N}(0,1)$ & 427.8 & $2.89 \pm 0.64 \pm 0.30$ & $\mathrm{GH}(0,6)$ & 387.7 & $7.6 \pm 1.6 \pm 0.5$ \\
\hline $1 \mathrm{~N}(1,1)$ & 388.5 & $3.9 \pm 1.7 \pm 0.3$ & & & \\
\hline
\end{tabular}

Table 4

Collisional quenching reference pressures in dry air at $293 \mathrm{~K}$. The quoted uncertainties are explained in the text.

\section{Application to the fluorescence detection of ultra high energy cosmic rays}

The number of fluorescence photons emitted at a given stage of a cosmic ray shower development, i.e. at a given altitude $h$ in the atmosphere, is given by:

$$
N_{\lambda}^{\text {shower }}(h)=E_{\text {dep }}^{\text {shower }}(h) Y_{\text {air }}(\lambda, p, T),
$$

where $E_{\text {dep }}^{\text {shower }}(h)$ is the energy deposited by the shower charged particles in the air volume, $p$ and $T$ are the air pressure and temperature at the altitude $h$. Notice that we assumed in Eq. (18) that the fluorescence emission is proportional to the energy deposited in the gas, which has been tested experimentally with good precision [10] 12 [38.

Fluorescence telescopes measure $N_{\lambda}^{\text {shower }}(h)$ to detect ultra high energy cosmic rays. If the fluorescence yield $Y_{\text {air }}(\lambda, p, T)$ is known, the energy deposited by the cosmic ray shower can be determined. Integrating along the shower path in the atmosphere will give the electromagnetic energy of the shower. The fluorescence efficiency must thus be known over a range of pressure and temperature corresponding to altitudes up to several tens of $\mathrm{km}$. 
It is convenient to express the fluorescence efficiency in terms of the physical quantities measured in this paper, namely the air fluorescence spectrum relative band intensities $I_{\lambda}\left(p_{0}, T_{0}\right)$ (Section 4) and the quenching reference pressures $p_{\text {air }}^{\prime}\left(\lambda, T_{0}\right)$ (Sections [5,6). The air pressure $p_{0}=800 \mathrm{hPa}$ and temperature $T_{0}=293 \mathrm{~K}$ used in the measurements have been introduced in the notation for consistency with what will be used in the following. The quenching reference pressure (cf. Eq. (5) ) can be written in a general way as:

$$
p_{\text {air }}^{\prime}(\lambda, T)=\frac{\sqrt{T}}{A_{\lambda} H_{\lambda}(T)},
$$

where $H_{\lambda}(T)$ has been introduced to take into account a possible temperature dependence of the collisional cross sections. In terms of the measured quenching reference pressures $p_{\text {air }}^{\prime}\left(\lambda, T_{0}\right)$, Eq. (19) becomes:

$$
p_{\text {air }}^{\prime}(\lambda, T)=p_{\text {air }}^{\prime}\left(\lambda, T_{0}\right) \sqrt{\frac{T}{T_{0}}} \frac{H_{\lambda}\left(T_{0}\right)}{H_{\lambda}(T)} .
$$

The fluorescence yield can thus be expressed in terms of the measured physical quantities:

$$
Y_{\text {air }}(\lambda, p, T)=Y_{\text {air }}\left(337, p_{0}, T_{0}\right) I_{\lambda}\left(p_{0}, T_{0}\right) \frac{1+\frac{p_{0}}{p_{\text {air }}^{\prime}\left(\lambda, T_{0}\right)}}{1+\frac{p}{p_{\text {air }}^{\prime}\left(\lambda, T_{0}\right) \sqrt{\frac{T}{T_{0}} \frac{H_{\lambda}\left(T_{0}\right)}{H_{\lambda}(T)}}}},
$$

This parameterization, together with the values of Tables 1, 4, and 5, can be used by ultra high energy cosmic rays experiments which employ the fluorescence detection technique. In Table 5 our best estimates for the unmeasured quenching reference pressure of the nine weak bands not present in Table 4 are reported. We assumed in Table 5 that all the Gaydon-Herman bands have the same $p^{\prime}$ of the $\mathrm{GH}(0,4)$ band. For the unmeasured $p^{\prime}$ of the $2 \mathrm{P}(3, \mathrm{i})$ and $2 \mathrm{P}(4, \mathrm{i})$ bands we took the average value of the measured $2 \mathrm{P}(3, \mathrm{i}=1,3)$ with a large uncertainty. The quenching reference pressure of the $1 \mathrm{~N}(1,2)$ was taken to be equal to the measured $p^{\prime}$ of the $1 \mathrm{~N}(1,1)$.

Notice that in order to fully determine the fluorescence yield, the absolute fluorescence yield of the $337 \mathrm{~nm}$ band, $Y_{\text {air }}\left(337, p_{0}, T_{0}\right)$, and the temperature dependence of the collisional cross sections, $H_{\lambda}(T)$ must be known. Measurements of these quantities will be the subject of subsequent papers.

The uncertainties on the relative band intensities and quenching reference pressures will result in an uncertainty on the cosmic ray shower energy. For a proper estimate of this uncertainty, the spectral sensitivity of the fluorescence detector and the wavelength dependent atmospheric attenuation must 


\begin{tabular}{|c|c|c||c|c|c|}
\hline Band & $\lambda(\mathrm{nm})$ & $p_{\text {air }}^{\prime}(\lambda)(\mathrm{hPa})$ & Band & $\lambda(\mathrm{nm})$ & $p_{\text {air }}^{\prime}(\lambda)(\mathrm{hPa})$ \\
\hline $2 \mathrm{P}(4,4)$ & 326.8 & $19 \pm 5 \pm 1$ & $\mathrm{GH}(6,2)$ & 302.0 & $21 \pm 10 \pm 1$ \\
$2 \mathrm{P}(4,7)$ & 385.8 & $19 \pm 5 \pm 1$ & $\mathrm{GH}(5,2)$ & 308.0 & $21 \pm 10 \pm 1$ \\
\cline { 1 - 3 } $2 \mathrm{P}(3,5)$ & 367.2 & $19 \pm 5 \pm 1$ & $\mathrm{GH}(6,3)$ & 317.6 & $21 \pm 10 \pm 1$ \\
$2 \mathrm{P}(3,7)$ & 414.1 & $19 \pm 5 \pm 1$ & $\mathrm{GH}(0,5)$ & 366.1 & $21 \pm 10 \pm 1$ \\
\hline $1 \mathrm{~N}(1,2)$ & 423.6 & $3.9 \pm 1.7 \pm 0.3$ & \multicolumn{4}{|c}{} \\
\hline
\end{tabular}

Table 5

Collisional quenching reference pressures in air at $293 \mathrm{~K}$ adopted for the nine weak bands for which $p^{\prime}$ could not be measured.

be taken into account because they change the relative weights of the fluorescence spectrum bands. A detailed study of these effects goes beyond the scope of this paper. Nevertherless, an estimate may be obtained from the total fluorescence yield:

$$
Y_{\text {tot }}(p, T)=\sum_{\lambda} Y_{a i r}(\lambda, p, T)
$$

where the sum goes over the 34 wavelengths of Table1. In Eq. (22) we assumed $H_{\lambda}(T)=1$, i.e. collisional cross sections have no temperature dependence. From Eq. (18), the relative uncertainty on the energy deposited by the cosmic ray shower is equal to the relative uncertainty on the total fluorescence yield, $\sigma_{Y_{t o t}} / Y_{t o t}$. In Fig. 13, the relative uncertainty on the total fluorescence yield is shown as a function of altitude. The U.S. 1976 Standard Atmosphere [39] was used to calculate the pressure and temperature at a given altitude. In the calculation of $\sigma_{Y_{t o t}}$, the uncertainties on the relative intensities of the spectrum bands and the quenching reference pressures were treated as uncorrelated, with the exception of the uncertainties related to $p_{\text {air }}^{\prime}(337)$ which were fully correlated for all the reference quenching pressures. Ultra high energy cosmic rays deposit most of their energy well below $25 \mathrm{~km}$ altitude, which corresponds to a vertical slant depth of only $14 \mathrm{~g} / \mathrm{cm}^{2}$. Thus, we may estimate from Fig. 13 that the uncertainties on the measurements reported in this work will result in a relative uncertainty on the cosmic ray shower energy at the level of $1 \%$.

From our knowledge of the spectrograph's calibration procedures and several checks performed, we estimate that the systematic uncertainty $(3.5 \%)$ on the relative intensities of the spectrum bands are largely uncorrelated. Even taking half of this uncertainty as fully correlated when calculating $\sigma_{Y_{t o t}}$, the relative uncertainty on the total fluorescence yield would still be very small, of the order of $2 \%$.

The AIRFLY fluorescence spectrum is significantly more precise than previous measurements, which had coarser spectrograph resolution [7] or made use of 


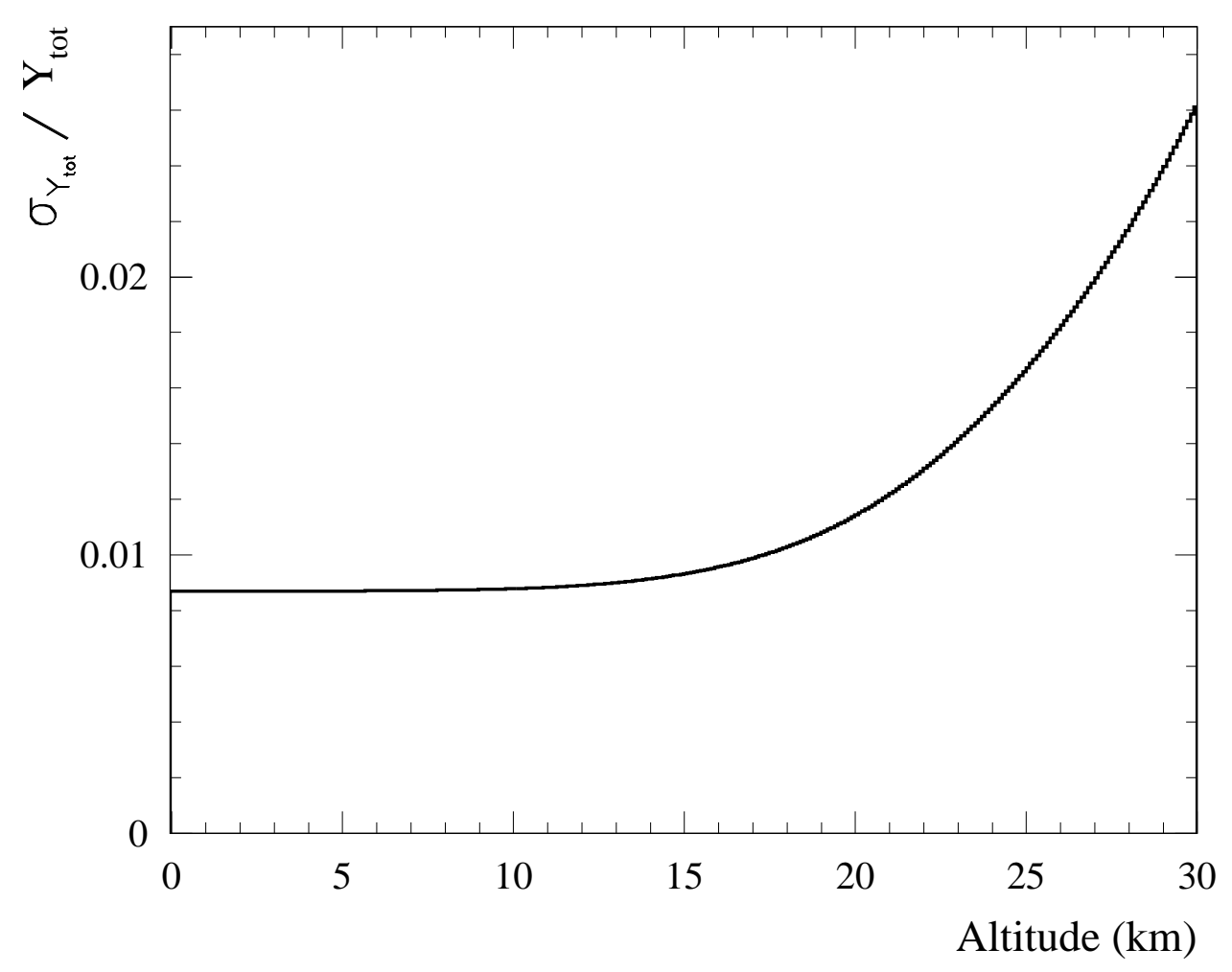

Fig. 13. Relative uncertainty on the total fluorescence yield as a function of altitude.

narrow band optical filters [9] [10]. For the purpose of comparison, the total fluorescence yield in the range 300 to $400 \mathrm{~nm}$ can be used, since this quantity was measured also by [11] and [12] with a broad band optical filter.

The ratio of the total fluorescence yield in the range 300 to $400 \mathrm{~nm}$ as measured by other experiments to the one measured by AIRFLY is shown as a function of altitude in Fig. 14. All yields were normalized to have the same value at ground level. Note that optical atmospheric attenuation and scattering effects have not been included in Fig. 14. Thus, the impact on the primary cosmic ray energy cannot be directly derived from this figure. Nevertheless, the total yields of Bunner [7] and Belz et al. [11] are in very good agreement with AIRFLY. Notice that the measurement of Belz et al. should not suffer from systematic uncertainties due to secondary electrons, since they measured the pressure dependence of the fluorescence lifetime. Kakimoto et al. 9], Nagano et al. 10] and Colin et al. [12] present significant deviations, which reflect the systematically higher values of $p^{\prime}$ measured by these experiments. 


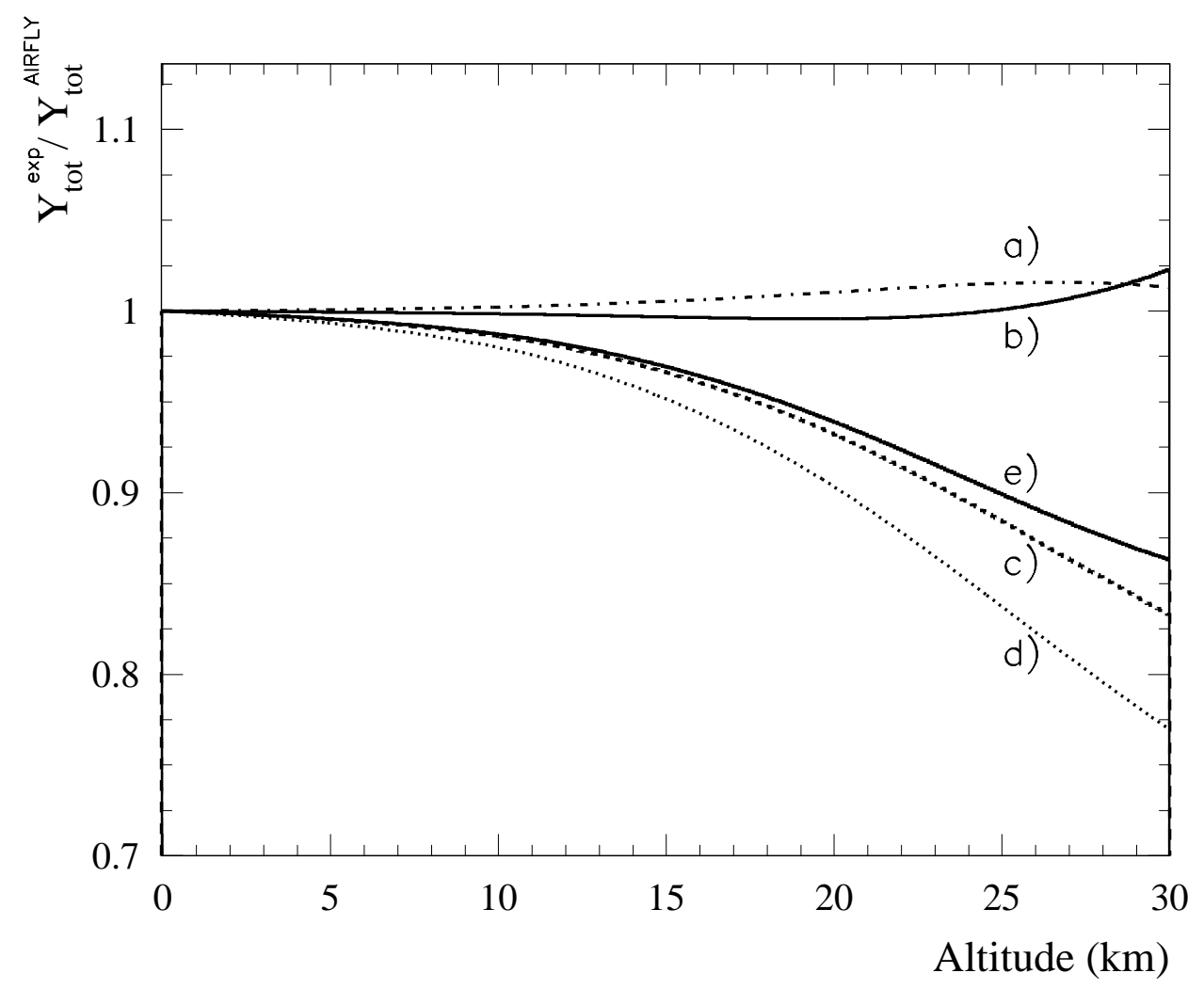

Fig. 14. Ratio of the total fluorescence yield in the range 300 to $400 \mathrm{~nm}$ as measured by other experiments to the one measured by AIRFLY as a function of altitude: a) Belz et al. b) Bunner c) Nagano et al. d) Kakimoto et al. e) Colin et al.

\section{Summary}

We have made a precise measurement of the emission spectrum of nitrogen molecules excited by $3 \mathrm{MeV}$ electrons in dry air. We could measure 34 fluorescence bands in the wavelength range from 284 to $429 \mathrm{~nm}$. The $2 \mathrm{P}$ and $1 \mathrm{~N}$ systems of molecular nitrogen were found to dominate the fluorescence emission. The high resolution spectrograph allowed the identification of a group of weaker bands, which was found to be consistent with the Gaydon-Herman bands. The relative intensities of bands corresponding to transitions from a common upper level were in good agreement with theoretical expectations based on the ratio of Einstein coefficients.

The pressure dependence of the fluorescence spectrum was also measured from a few hPa up to atmospheric pressure. Particular care was taken to avoid the bias from undetected light due to secondary electrons escaping the detector's field of view at low pressures. For this purpose, the relative intensity of the $337 \mathrm{~nm}$ band was measured as a function of pressure in both nitrogen and air, 
and from the ratio of intensities measured with the two gases, a measurement of the collisional quenching reference pressure, $p_{\text {air }}^{\prime}(337)$, was obtained. The measurement of the fluorescence spectrum at different pressures allowed the determination of the collisional quenching reference pressures of 24 fluorescence bands, in addition to the $337 \mathrm{~nm}$ band. Systematic uncertainties were minimized by taking the intensities relative to the $337 \mathrm{~nm}$ band, and by using the value of $p_{\text {air }}^{\prime}(337)$ previously measured. Consistent values of the collisional quenching reference pressures were found for bands due to transitions from a common upper level.

The effect of argon on the fluorescence yield was also investigated, and found to be negligible.

The high resolution spectra recorded by the spectrograph allowed many more closely spaced bands to be separated than in previous experiments. In the ratio of fluorescence intensities, which was extensively used, many systematic uncertainties cancel. Thanks to these improvements in the experimental method, the details and precision of the AIRFLY measurements surpass that of previous experiments. The application of the AIRFLY results to ultra high energy cosmic ray experiments which employ the fluorescence detection technique was also studied. A parameterization of the fluorescence yield as a function of altitude in terms of the measured relative band intensities and collisional quenching pressures was derived. We estimated that the systematic uncertainty on the cosmic ray shower energy associated with the pressure dependence of the fluorescence spectrum is reduced to a level of $1 \%$ by the AIRFLY results presented in this paper.

\section{Acknowledgements}

The authors wish to thank M. Iannilli, D. Pecchi, L. Sdogati, E. Tusi and G. Vitali of Università di Roma Tor Vergata for the assistance in the construction of the pressure chamber, Ilya A. Shkrob and R.H. Lowers of the Argonne Chemistry Division for their support and assistance with the Van de Graaff maintenance and operations. This work was supported by the following agencies and organizations: United States Department of Energy, Office of Science, Offices of High Energy Physics and Basic Energy Sciences, under contract DE-AC02-06CH11357; BMBF grant no. 05 CU5VK1/8 and DFG grant no. KE 1151/1; project GA CR no. 202/05/2470 and project MSMT INGO; Istituto Nazionale di Fisica Nucleare, Italy; Center of excellence CETEMPS

of Università de l'Aquila. 


\section{References}

[1] R.M. Baltrusaitus et al., Nucl. Instrum. Meth. Phys. Res. A 240 (1985) 410.

[2] T. Abu-Zayyad et al., Nucl Instrum. Meth. Phys. Res. A 450 (2000) 253.

[3] J. Abraham et al., Nucl Instrum. Meth. Phys. Res. A 523 (2004) 50.

[4] M. Fukushima, Prog. Theor. Phys. Suppl. 151 (2003) 206.

[5] L. Scarsi et al., Proc. 27th Int. Cosmic Ray Conf., Hamburg (2001) 839

[6] F. W. Stecker et al., Nucl. Phys. B136 (2004) 433

[7] A. N. Bunner, Cosmic Ray Detection by Atmospheric Fluorescence, PhD thesis, Graduate School of Cornell University, February 1967.

[8] G. Davidson and R. O'Neil, J. Chem. Phys. 41 (1964) 3946.

[9] F. Kakimoto et al., Nucl. Instrum. Meth. Phys. Res. A 372 (1996) 527.

[10] M. Nagano et al., Astropart. Phys. 20 (2003), 293; 22 (2004), 235.

[11] J. W. Belz et al., Astropart. Phys. 25 (2006) 129.

[12] P. Colin et al., astro-ph/06121110, to appear in Astropart. Phys.

[13] V. de Souza, G. Medina-Tanco, and J. A. Ortiz, Astropart. Phys. 25 (2006) 84.

[14] B. Keilhauer et al., Astropart. Phys. 25 (2006) 259.

[15] F. Arqueros et al., Astropart. Phys. 26 (2006) 231.

[16] A. Lofthus and P. H. Krupenie, J. Phys. Chem. Ref. Data, 6 (1977) 113.

[17] K. B. Mitchell, J. Chem. Phys. 53 (1970) 1795

[18] J. M. Calo and R. C. Axtmann, J. Chem. Phys. 54 (1971) 1332

[19] High Voltage Engineering Corporation model KS Van de Graaff.

[20] An Eimac Y646 cathode electron source was used from Communications and Power Industries, Eimac Division, 607 Hansen Way, Palo Alto CA 94304 USA.

[21] M. Conde et al., Proceedings of Particle Accelerator Conference, PAC05, Knoxville, Tennessee, USA, May 16-20,2005, TPAE016.

[22] Bergoz Instrumentation, Espace Allondon Ouest, 156, rue du Mont Rond, 01630 Saint Genis Pouilly, France, model ICT-088-070-20:1.

[23] Hamamatsu Photonics K. K., 314-5, Shimokanzo, Toyooka-village, Iwata-gun, Shizuoka-ken, 438-0193, Japan.

[24] Varian, Inc., 121 Hartwell Avenue, Lexington, MA 02421, model SH01001UNIV. 
[25] Oerlikon Leybold Vacuum GmbH, Bonner Strasse 498, Cologne D-50968, Germany, model Ceravac CTR 90.

[26] Spectra-Physics, 150 Long Beach Boulevard, Stratford, Ct 06615, USA.

[27] Andor Technology PLC, 7 Millenium Way, Springvale. Business Park, Belfast, BT12 7AL, Northern Ireland, model DV420 - BU2 CCD camera.

[28] National Institute of Standards and Technology, Gaithersburg, MD, 20899, USA

[29] A. R. Filippelli, S. Chung, and C. C. Lin, Phys. Rev. A29 (1984) 1709.

[30] D. J. Eckstrom, J. S. Dickinson, and R. M. Hill, J. Chem. Phys. 82 (1985) 1792.

[31] F. R. Gilmore, R. R. Laher, and P. J. Espy, J. Phys. Chem. Ref. Data, 21 (1992) 1005.

[32] J. T. Fons, R. S. Schappe, and C. C. Lin, Phys. Rev. A53 (1996) 2239.

[33] W. L. Borst and. M. Imami, J. Appl. Phys. 44 (1973) 1133.

[34] F. Blanco. and F. Arqueros, Phys. Lett. A345 (2005) 355

[35] R. M. Sternheimer, S. M. Seltzer, and M. J. Berger, Phys. Rev. B26 (1982), 6067 .

[36] S. Agostinelli et al., Nucl. Instrum. Meth. A506 (2003) 250.

[37] J. Baro' et al., Nucl. Instrum. Meth. B100 (1995) 31; F. Salvat, J. M. FernandezVarea, and J. Sempau, Workshop Proceedings, Barcelona, Spain, 4-7 July 2006, NEA\#06222, ISBN: 92-64-02301-1.

[38] J. Belz et al., Astropart. Phys. 25 (2006) 57.

[39] U.S. Standard Atmosphere 1976, U.S. Government Printing Office (Washington, D.C., 1976). 